\title{
Quality of patients' files documented by medical students and physicians at Tohid Hospital in Sanandaj
}

Asefeh Abbaszadeh ${ }^{1}$, Khaled Rahmani ${ }^{2}$, BaharMoasses-Ghafari ${ }^{3}$, Asrin Babahajian ${ }^{4}$

1. Assistant Professor of Infectious Diseases, School of Medicine, Kurdistan University of Medical Sciences, Sanandaj, Iran. ORCID ID: 0000-0002-0313-4632

2. Assistant Professor of Epidemiology, Liver and Digestive Research Center, Research Institute for Health Development, Kurdistan University of Medical Sciences, Sanandaj, Iran. ORCID ID: 0000-0002-0860-8040

3. Assistant Professor of Nuclear Medicine, School of Medicine, Kurdistan University of Medical Sciences, Sanandaj, Iran. (Corresponding Author), Tel: 087-33664645, E-mail: baharmoassesghafari@ gmail.com,ORCID ID: 0000-0001-5288-6243

4. MSc, Liver and Digestive Research Center, Research Institute for Health Development, Kurdistan University of Medical Sciences, Sanandaj, Iran., ORCID ID: 0000-0003-0278-1560

\begin{abstract}
ABSTARCT
Background and Aim: Previous studies in Iran have shown that the quality of documentation of medical records by physicians and medical staff is not very satisfactory. The purpose of this study was to investigate the quality of medical records documentation in Tohid teaching hospital, , in Sanandaj, Iran.
\end{abstract}

Materials and Methods: In this descriptive-analytical study, medical records of 430 patients admitted to various services of Tohid Hospital in Sanandaj during the first six months of 2017 were examined. Variables were collected using a checklist based on the national regulations for the documentation of medical records. Data were analyzed using SPSS V.20 software and Chi-square test.

Results: In the present study we observed registration of the correct diagnosis, in accordance with the principles of diagnosis, in $261(60.7 \%)$ of admission forms and the time of first visit of the physician had been recorded in $282(66.0 \%)$ cases. More than half of the history sheets and most of the initial, daily, and final reports were incomplete. The professors had evaluated $133(40.7 \%), 76(22.0 \%)$ and $5(7.2 \%)$ history sheets completed by the residents, interns and externs respectively. About $563(66.2 \%)$ cases of the doctor's prescriptions had been properly recorded in the files. We found correct registration of medical orders by interns and residents and professors in $101(69.2 \%)$ and $186(87.7 \%)$ and $240(53.7 \%)$ cases respectively.

Conclusion: Based on the findings of this study, it is necessary to hold training courses on proper documentation of medical records for physicians and medical students and more precise supervision of professors and physicians on performance of the medical students in all educational hospitals of Kurdistan University of Medical Sciences.

Keywords: Medical record, Health record, Hospital, Documentation

Received: May 26, 2020

Accepted: Oct 4, 2020

How to cite the article: Asefeh Abbaszadeh, Khaled Rahmani, BaharMoasses-Ghafari, Asrin Babahajian. Quality of patients' files documented by medical students and physicians at Tohid Hospital in Sanandaj. SJKU 2021;26(4):93-104.

Copyright (C) 2018 the Author (s). Published by Kurdistan University of Medical Sciences. This is an open access article distributed under the terms of the Creative Commons Attribution-Non Commercial License 4.0 (CCBYNC), where it is permissible to download, share, remix, transform, and buildup the work provided it is properly cited. The work cannot be used commercially without permission from the journal 


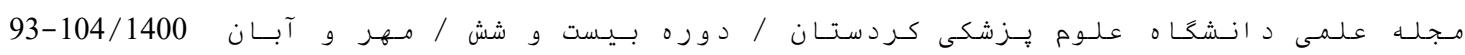

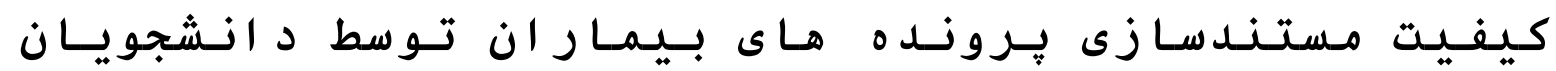

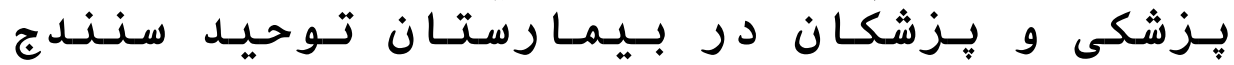

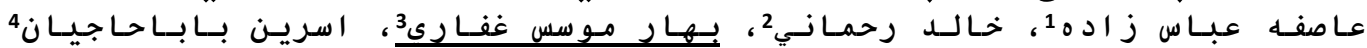

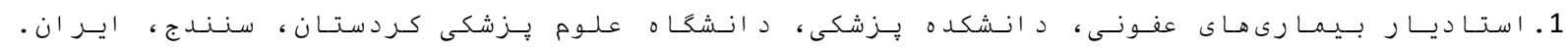

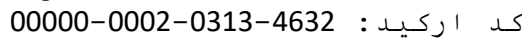

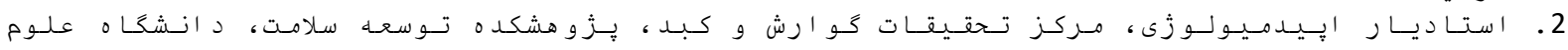

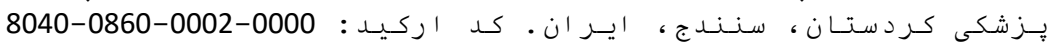

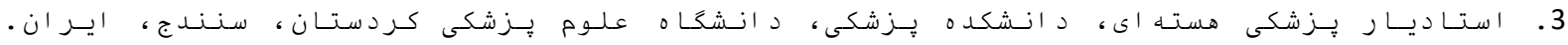

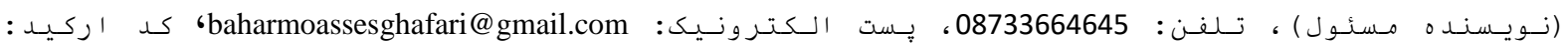
0000-0001-5288-6243

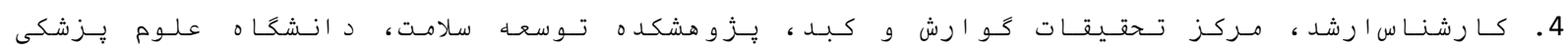

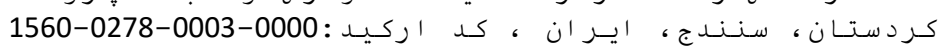

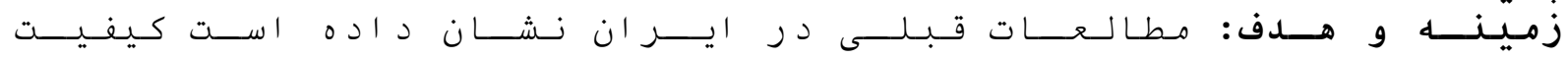

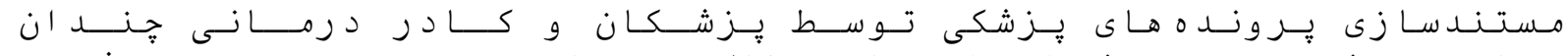

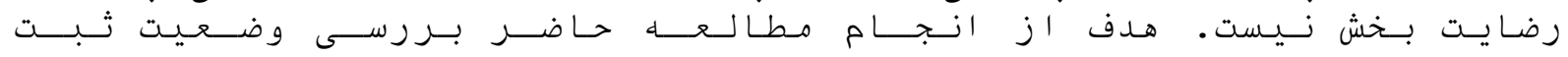

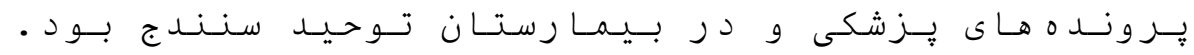

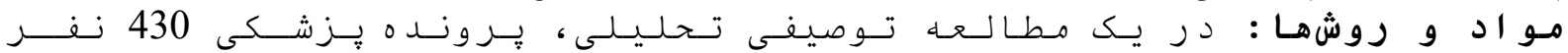

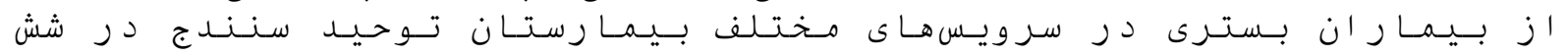

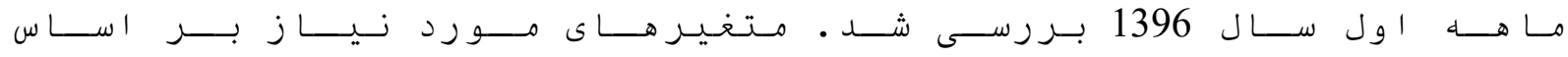

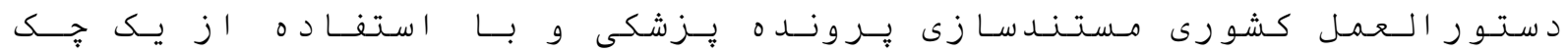

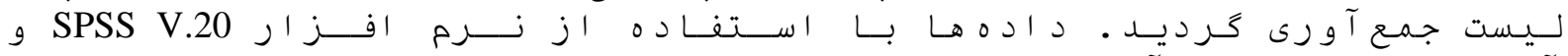

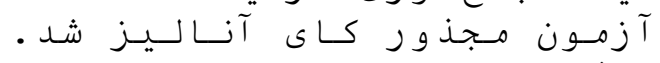

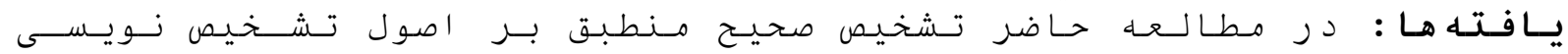

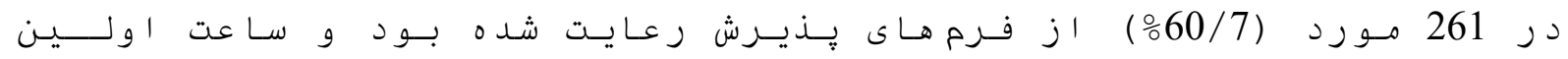

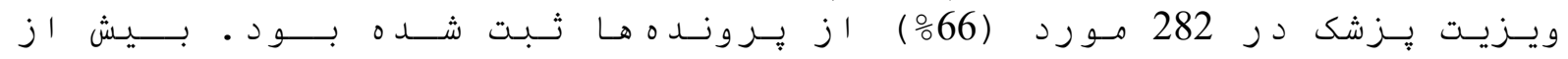

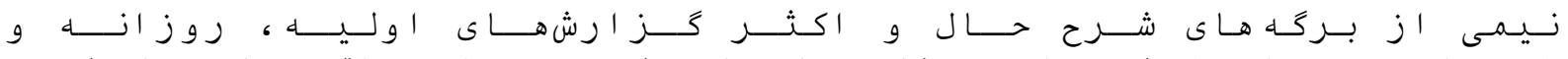

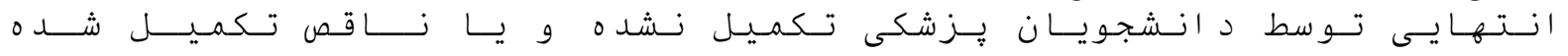

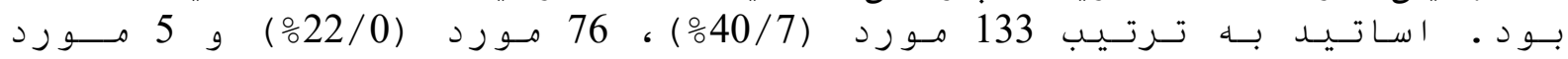
(7\%/2/2)

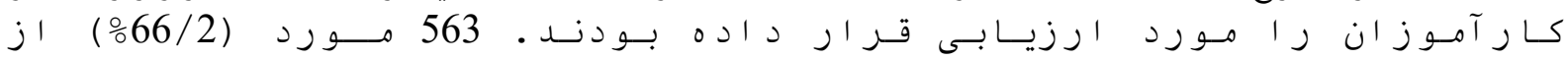

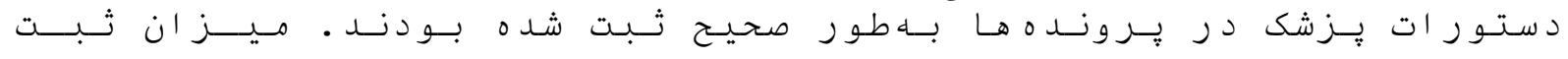

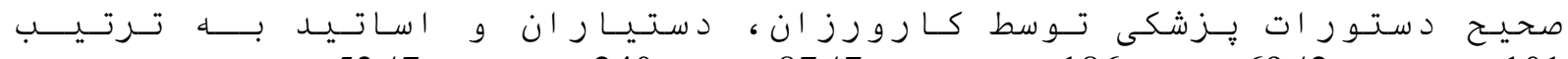

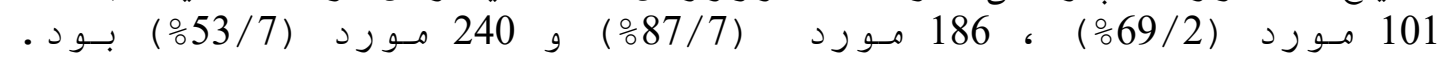

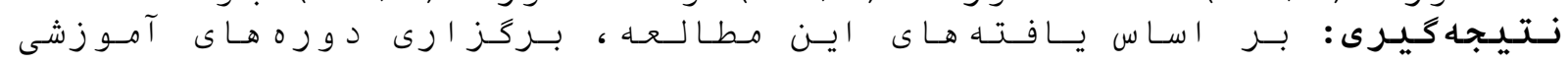

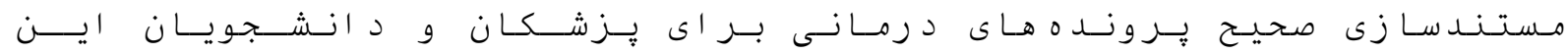

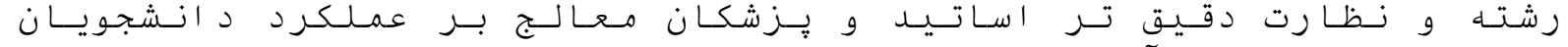

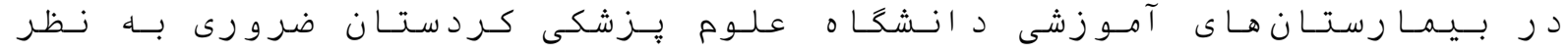

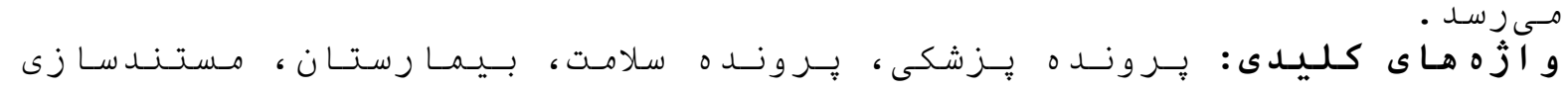

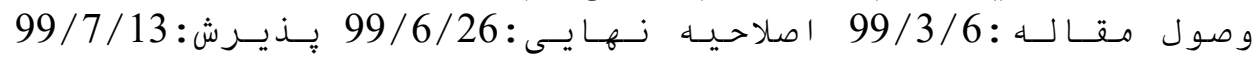




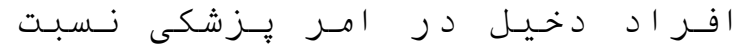

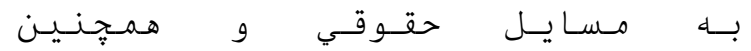

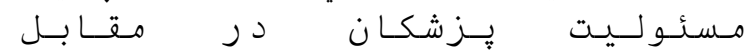

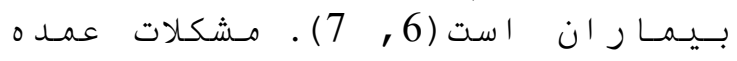

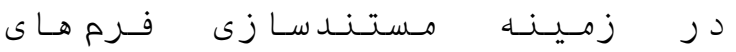

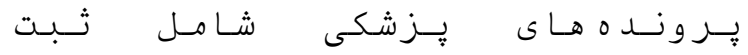

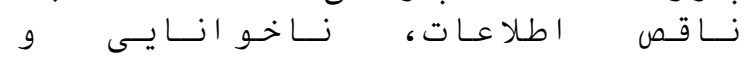

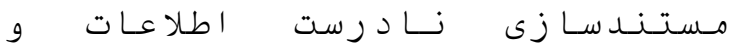

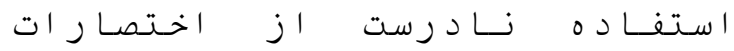

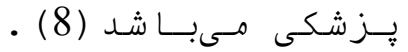

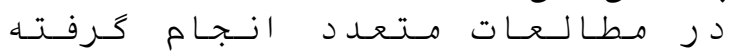

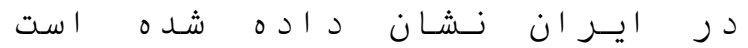

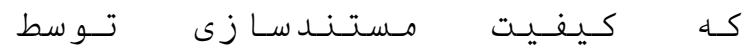

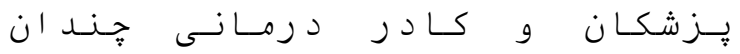

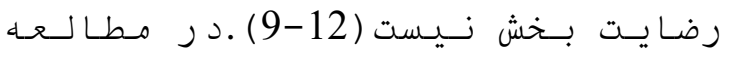

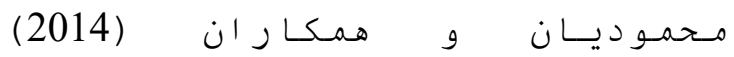

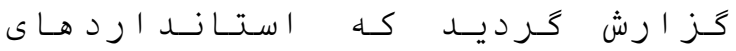

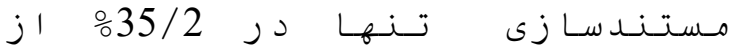

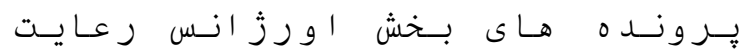

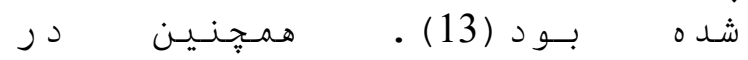

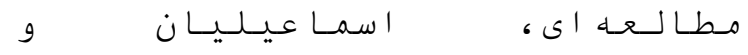

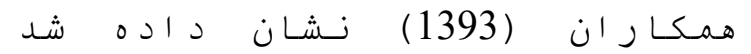

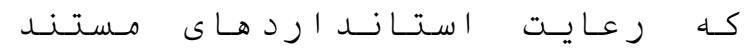

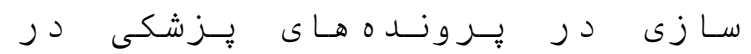

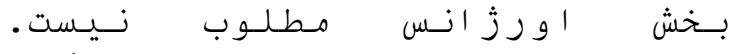

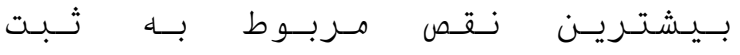

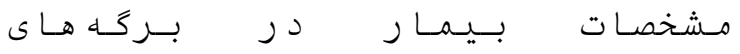

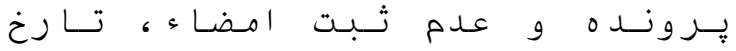

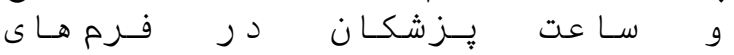

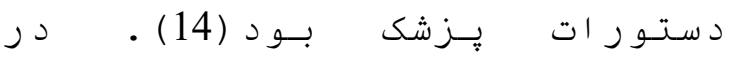

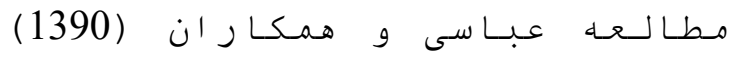

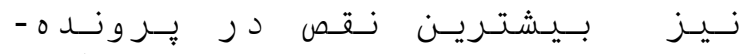

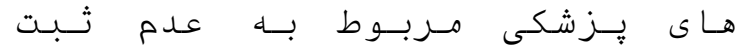

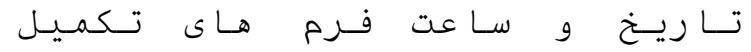

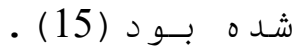

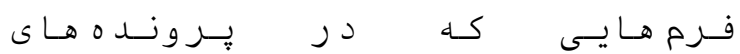

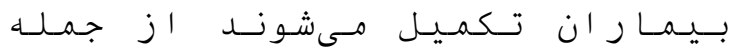

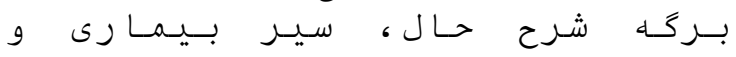

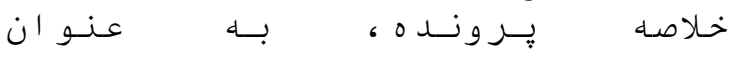

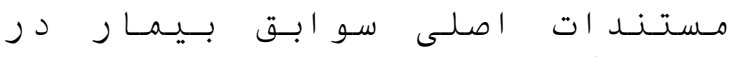

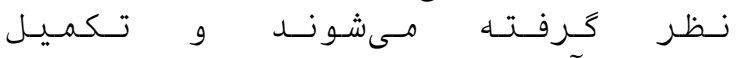

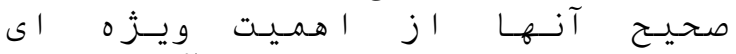

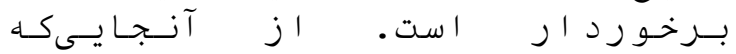

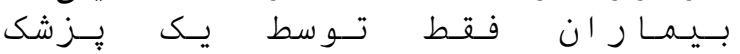

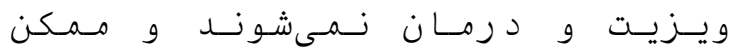

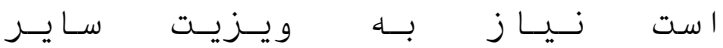

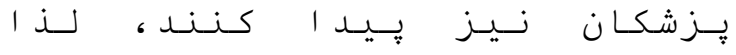

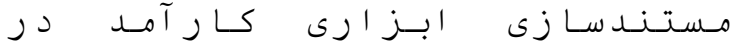

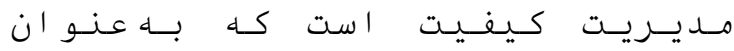

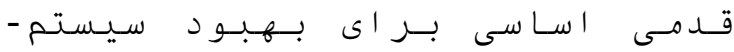

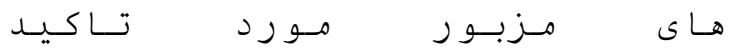

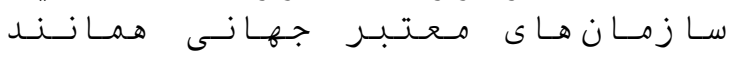

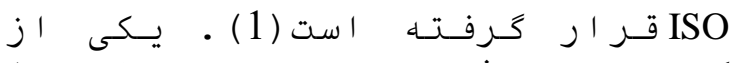

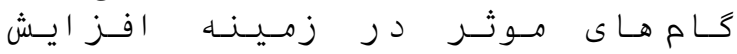

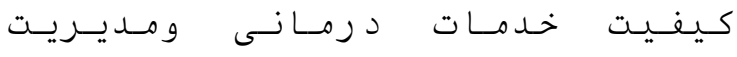

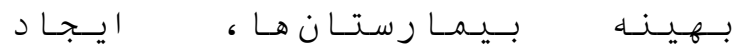

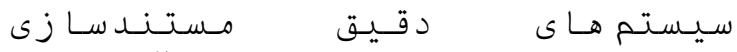

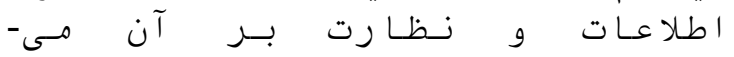

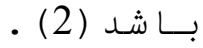

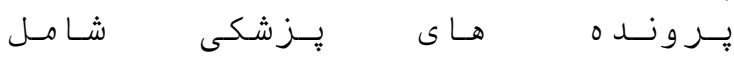

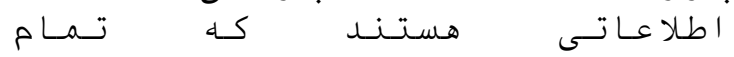

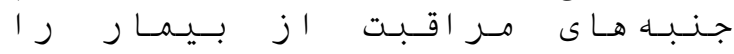

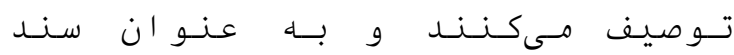

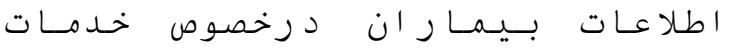

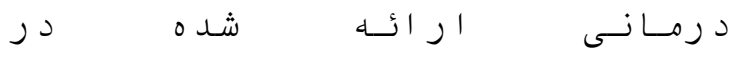

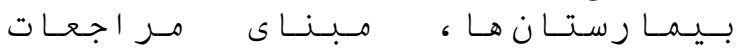

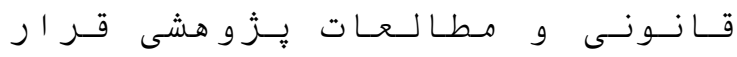

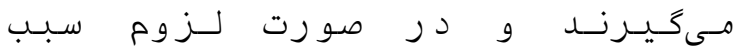

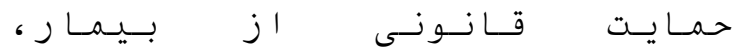

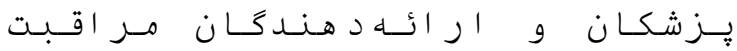

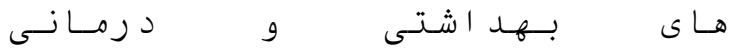

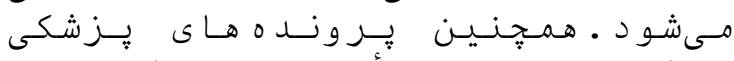

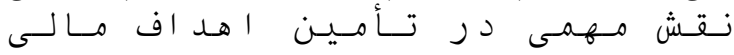

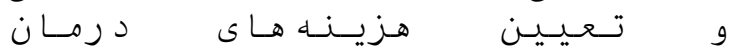

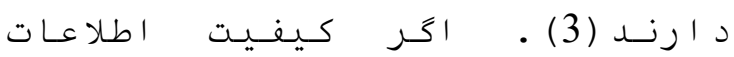

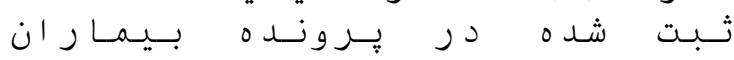

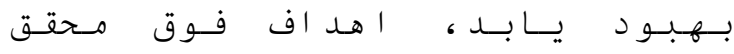

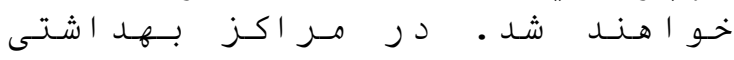

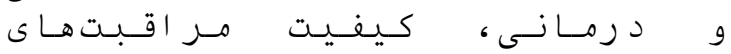

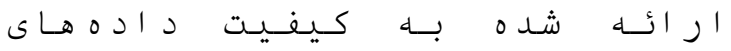

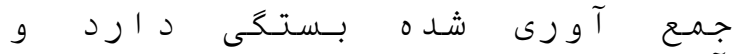

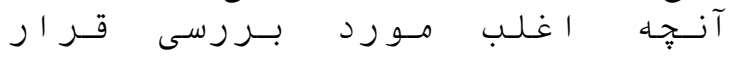

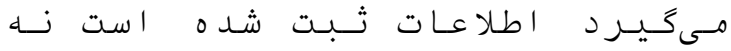

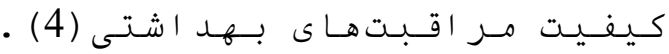

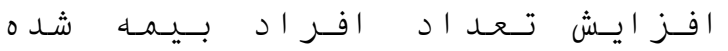

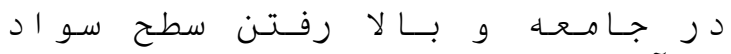

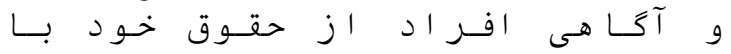

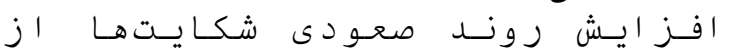

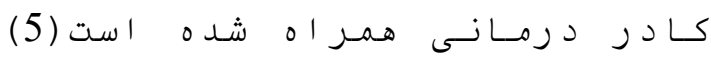

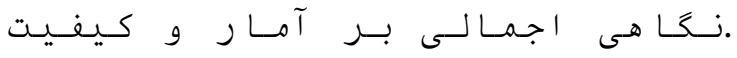

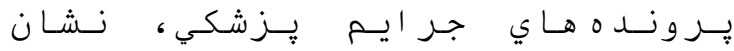

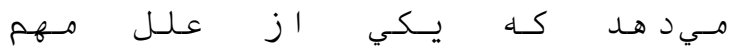

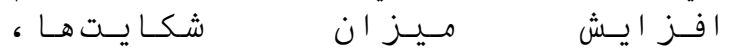

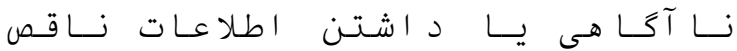

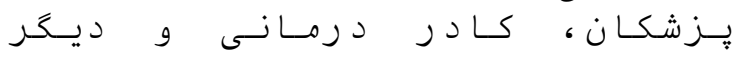




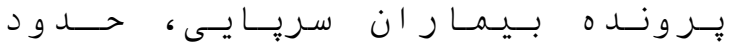

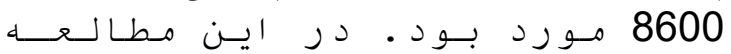

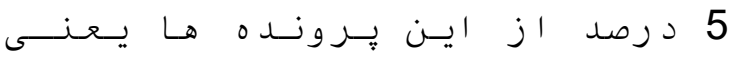

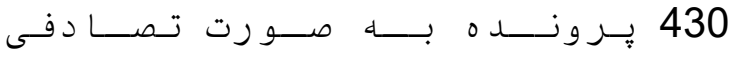

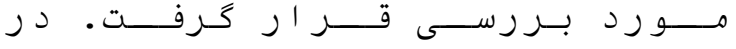

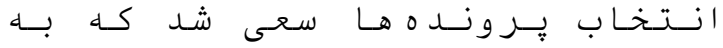

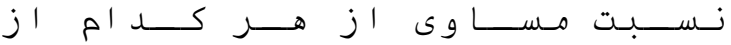

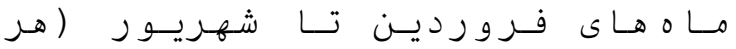

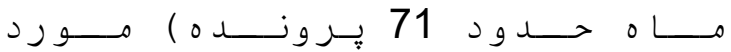

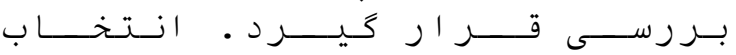

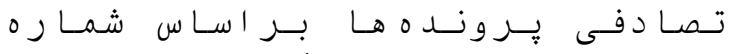

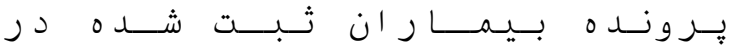

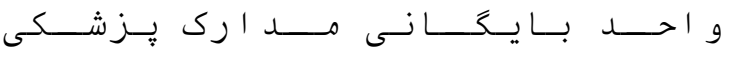

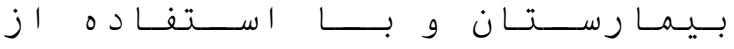

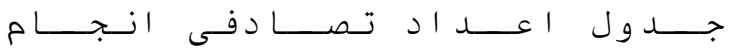

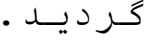

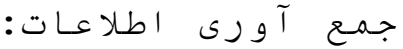

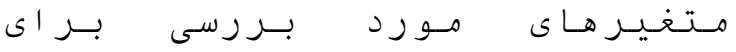

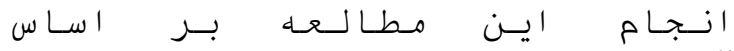

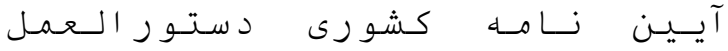

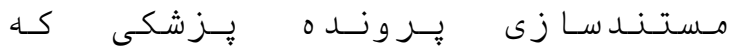

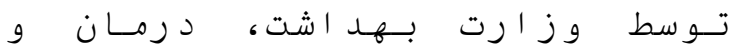

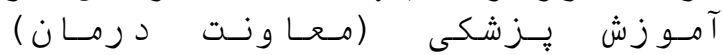

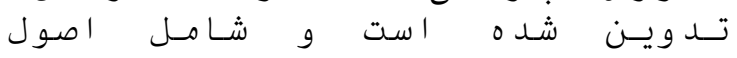

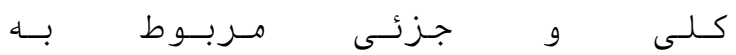

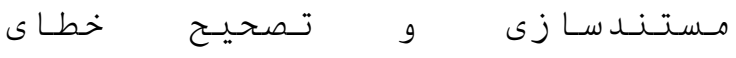

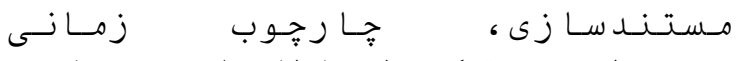

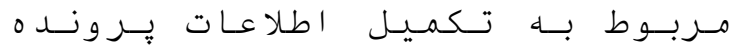

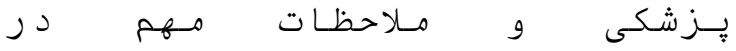

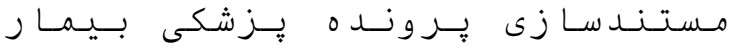

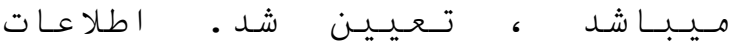

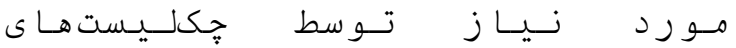

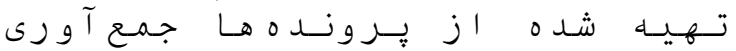

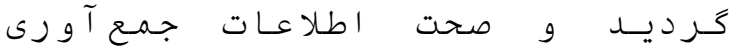

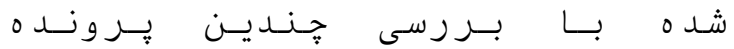

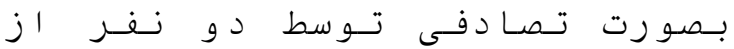

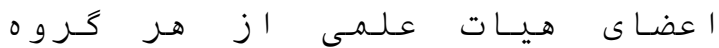

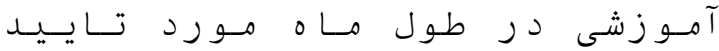

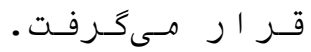

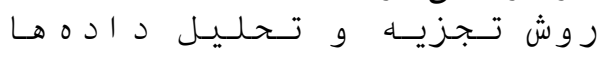

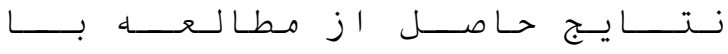

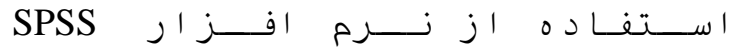

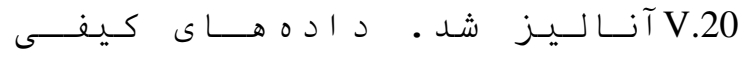

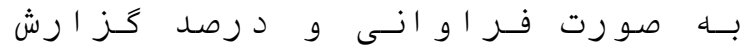

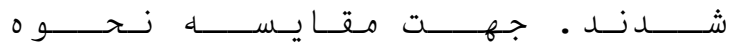

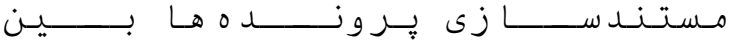

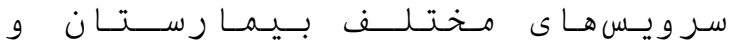

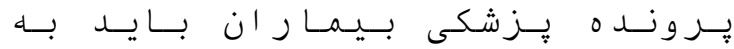

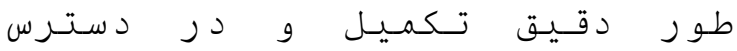

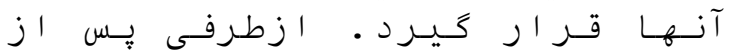

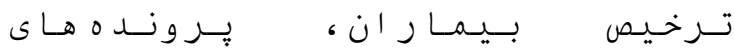

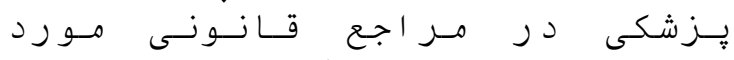

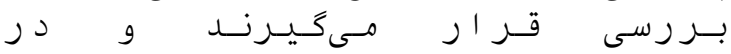

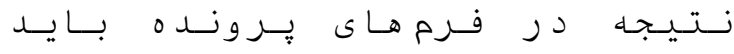

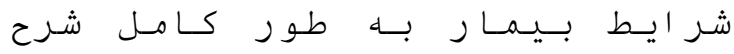

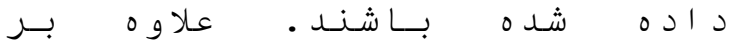

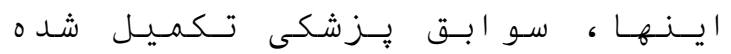

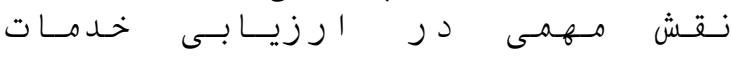

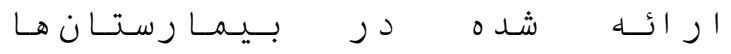

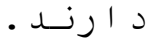

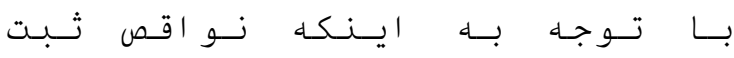

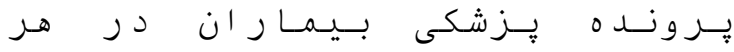

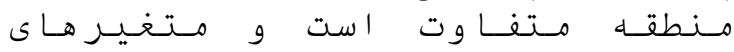

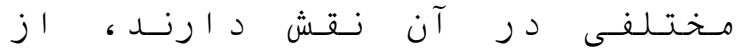

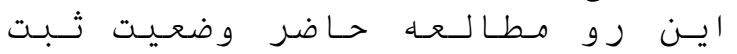

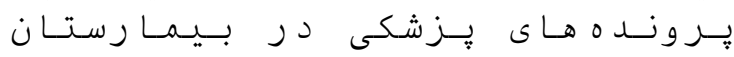

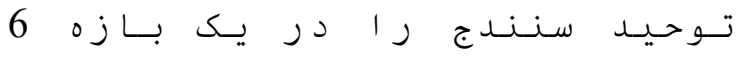

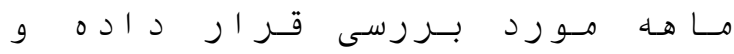

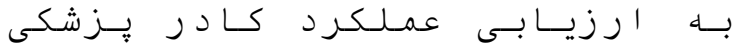

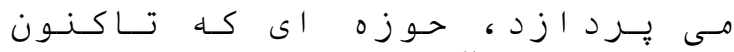

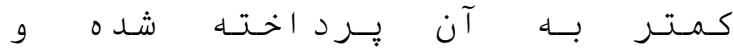

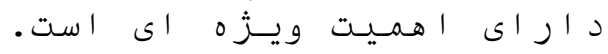

\section{مـو اد و رو روشهـا}

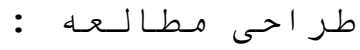

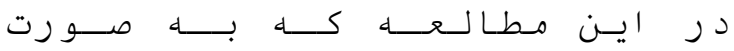

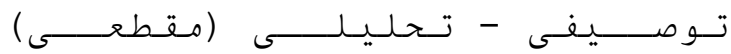

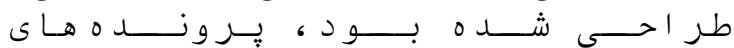

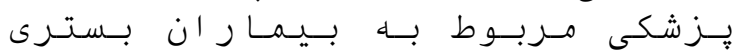

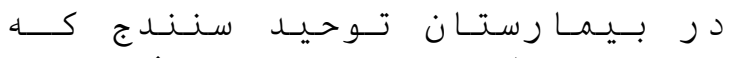

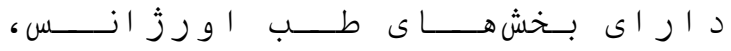

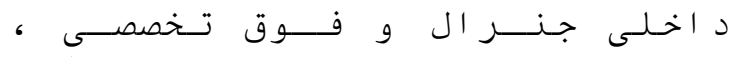

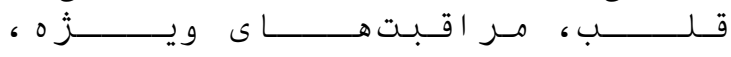

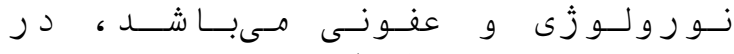

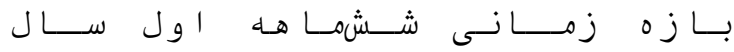

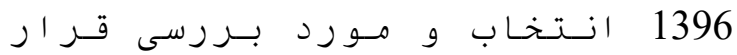

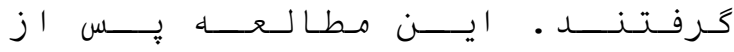

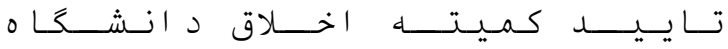

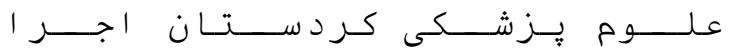

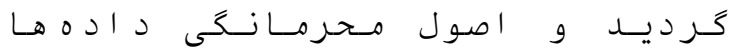

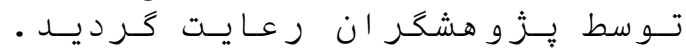

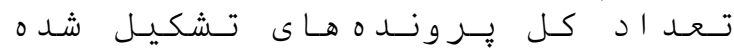

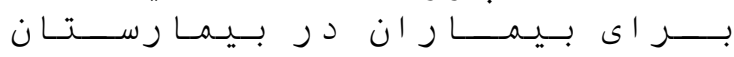

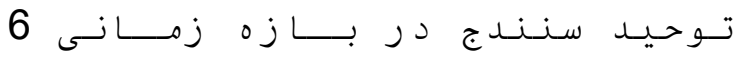

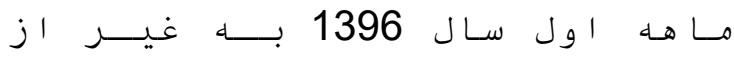




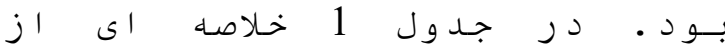

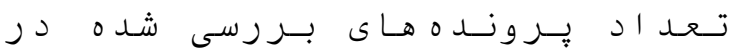

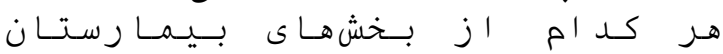

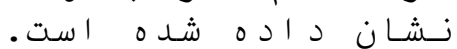

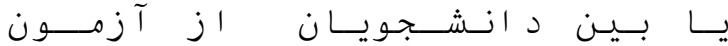

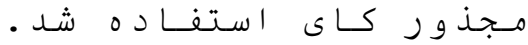

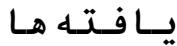

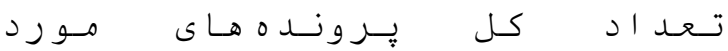

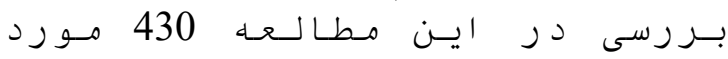

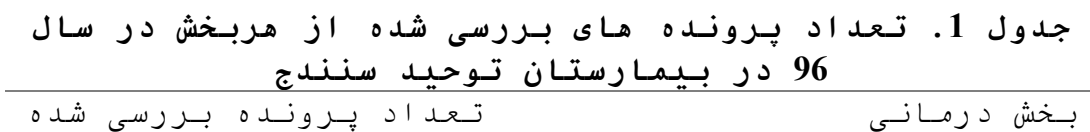

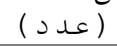

\begin{tabular}{|c|c|}
\hline 28 & عمدومس \\
\hline 23 & انـــــولـوز زى \\
\hline 3 & رو ومــاتــولــوزى زى \\
\hline 27 & ريــ \\
\hline 5 & مسلمـو مــــت \\
\hline 7 & غـد د \\
\hline 20 & كــــ ارش \\
\hline 6 & نـــــر ولــوزى \\
\hline 41 & \\
\hline 9 & $\mathrm{CCU}$ \\
\hline 28 & عفـونـى \\
\hline 23 & نــو رولـــوزى زي \\
\hline 12 & ICU \\
\hline 192 & طب اورز انـس \\
\hline 5 & جـر احى \\
\hline 1 & اوروولـــوزى \\
\hline 430 & مـجمـوع \\
\hline
\end{tabular}

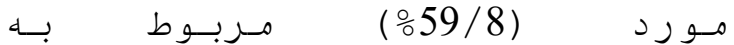

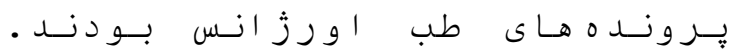

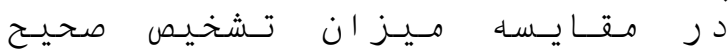

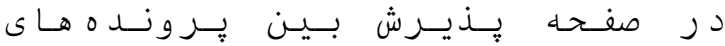

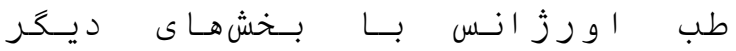

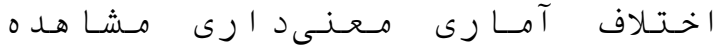

$$
\text { شـ ( }
$$

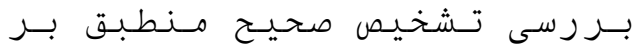

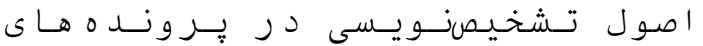

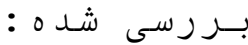

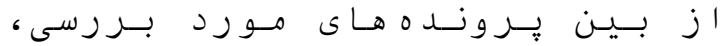
261

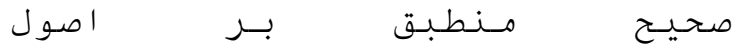

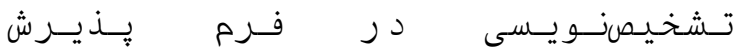

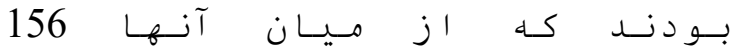

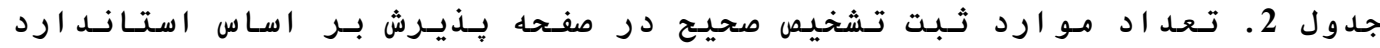

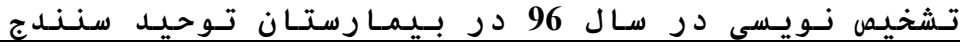

\begin{tabular}{|c|c|c|c|c|}
\hline Pvalue & اوزيـر انسب & طب اورز انسس & & \\
\hline & $105(44 \div / 1)$ & $156(81 \% / 2)$ & بــــ & 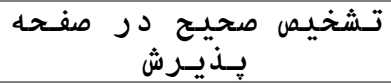 \\
\hline \multirow[t]{2}{*}{$<0 / 001$} & $133(55 \div / 9)$ & $36(18 \div / 8)$ & خيـر & \\
\hline & 238 & 192 & & مـجموع \\
\hline
\end{tabular}

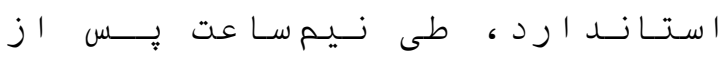

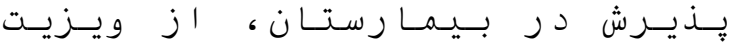

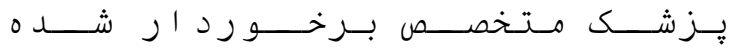

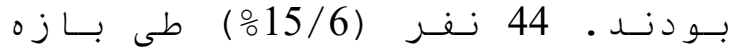

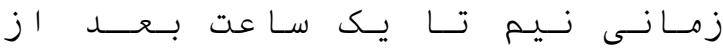

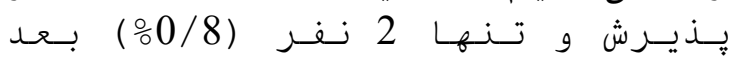

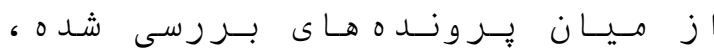

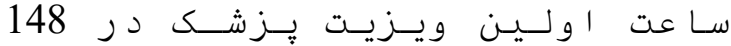

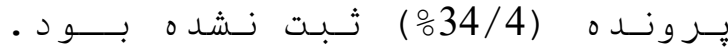

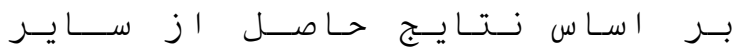

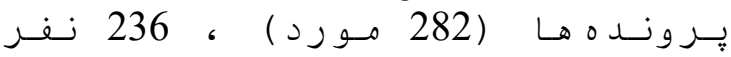

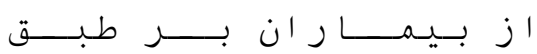

$(83 \% / 6)$ 


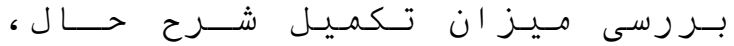

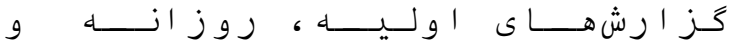

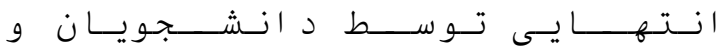

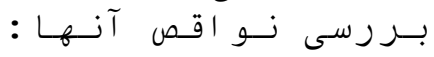

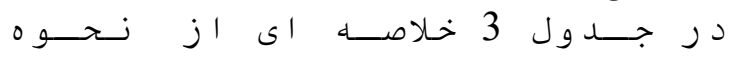

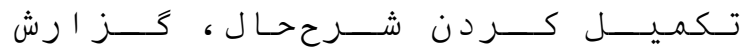

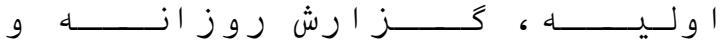

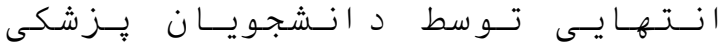

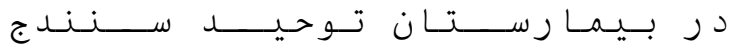

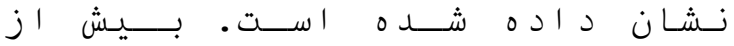

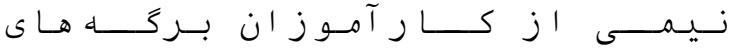

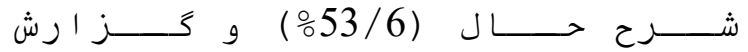

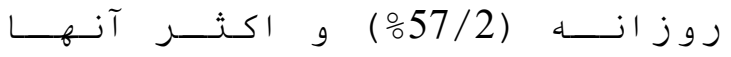

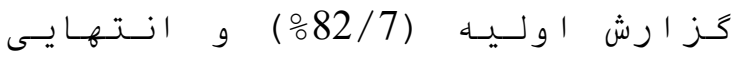

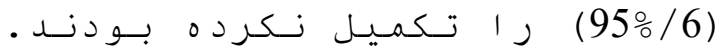

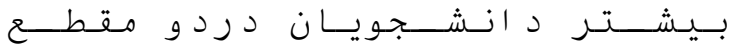

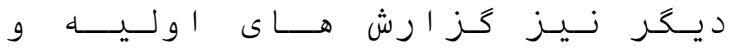

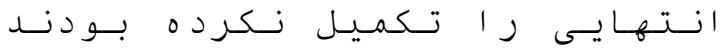

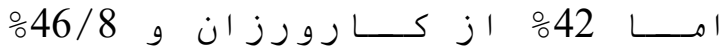

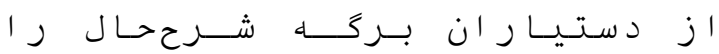

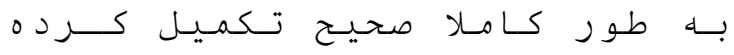

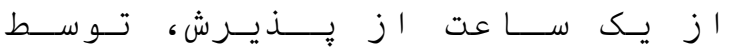

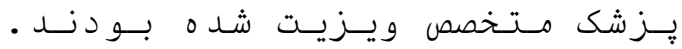

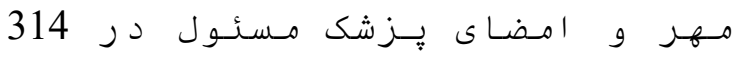

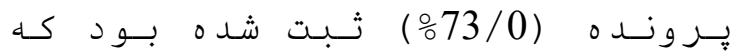
161

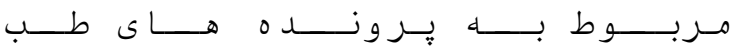

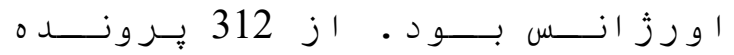

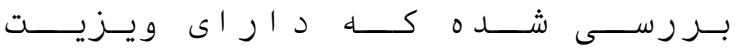

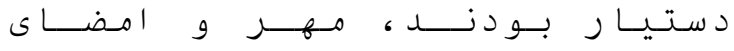

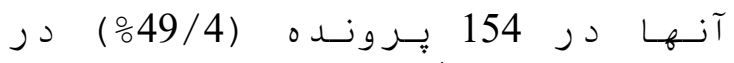

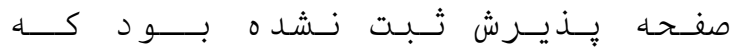

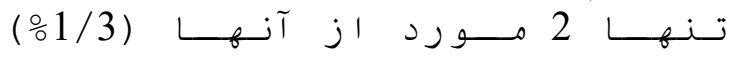

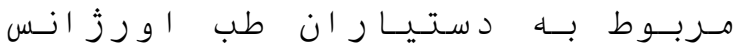

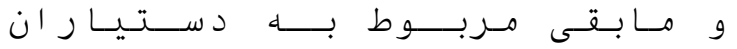

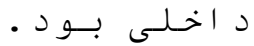

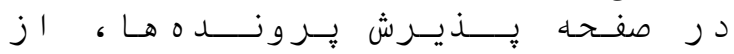

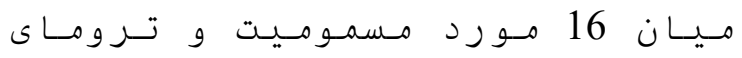

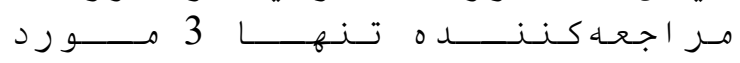

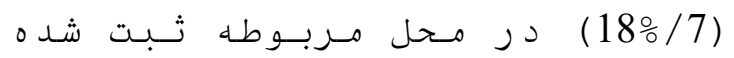

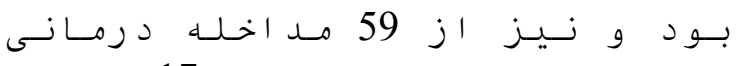

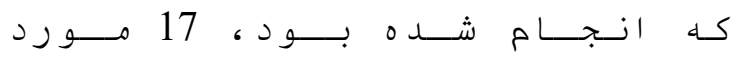

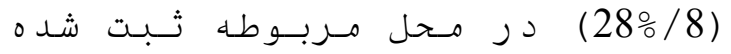

\begin{tabular}{|c|c|c|c|c|c|}
\hline \multirow[b]{2}{*}{ مجم-وع ع } & \multicolumn{3}{|c|}{ نـحوه تـكميـل } & & \multirow[t]{2}{*}{ 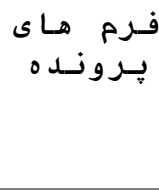 } \\
\hline & تـشـــيـل & نـــــ & كسامساح & & \\
\hline $69(100 \%)$ & $37(53 \div / 6)$ & $12(17 \% / 4)$ & $20(29 \div / 0)$ & كـار آمــوز & شرح حـال \\
\hline $345(100 \%)$ & $92(26 \div / 6)$ & $108(31 \% / 4)$ & $145(42 \div / 0)$ & كـارورز & \\
\hline $327(100 \%)$ & $82(25 \% / 1)$ & $\begin{array}{c}92 \\
(28 \% / 1) \\
\end{array}$ & $153(46 \div / 8)$ & د ستـيـا ر & \\
\hline $81(100 \%)$ & $67(82 \% / 7)$ & $10(12 \circ / 4)$ & $4(4 \div / 9)$ & كـار آمــوز & 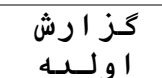 \\
\hline $180(100 \%)$ & $134(74 \div / 4)$ & $34(18 \div / 9)$ & $12(6 \div / 7)$ & كـارورز & \\
\hline $115(100 \%)$ & $96(83 \div / 5)$ & $7(6 \div / 1)$ & $12(10 \div / 4)$ & دســــــار ر & \\
\hline $84(100 \%)$ & $\begin{array}{c}(57 \% / 2) \\
48\end{array}$ & $\begin{array}{c}(35 \% / 7) \\
30\end{array}$ & $\begin{array}{c}\left(\frac{\circ}{\circ} 7 / 1\right) \\
6\end{array}$ & كـار رآمـوز ز & روز انــه اش \\
\hline $179(100 \%)$ & $\begin{array}{c}(42 \% / 4) \\
76\end{array}$ & $\begin{array}{c}(43 \% / 6) \\
78\end{array}$ & $\begin{array}{c}(14 \% / 0) \\
25\end{array}$ & كـا رو ر ز & \\
\hline $114(100 \%)$ & $\begin{array}{c}(51 \% / 7) \\
59\end{array}$ & $\begin{array}{c}(31 \% / 6) \\
36\end{array}$ & $\begin{array}{c}(16 \div / 7) \\
19\end{array}$ & د سـتيــا ر & \\
\hline $23(100 \%)$ & $22(95 \div / 6)$ & $1(4 \% / 4)$ & $0(0)$ & كـار آمــوز & كـز ارش \\
\hline
\end{tabular}




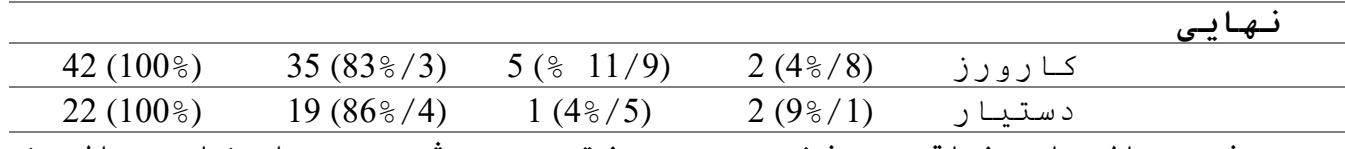

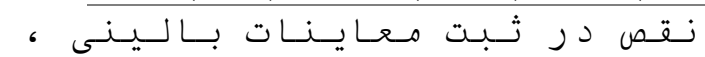

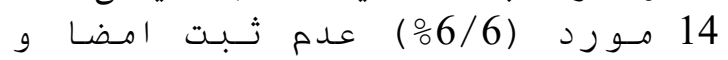

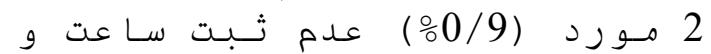

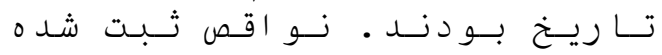

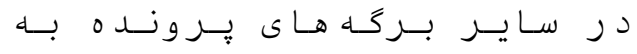

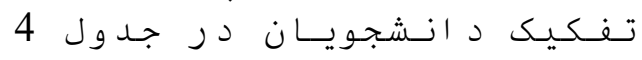

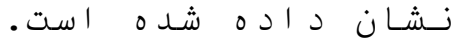

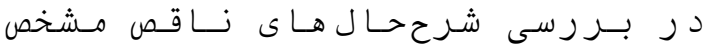

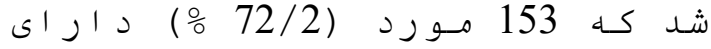

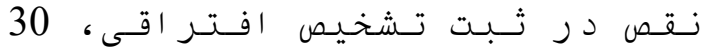

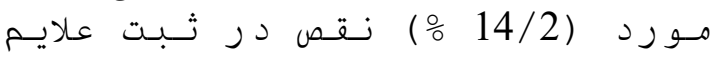
حيـاتـى ، 13 مــو رد د (14/2)

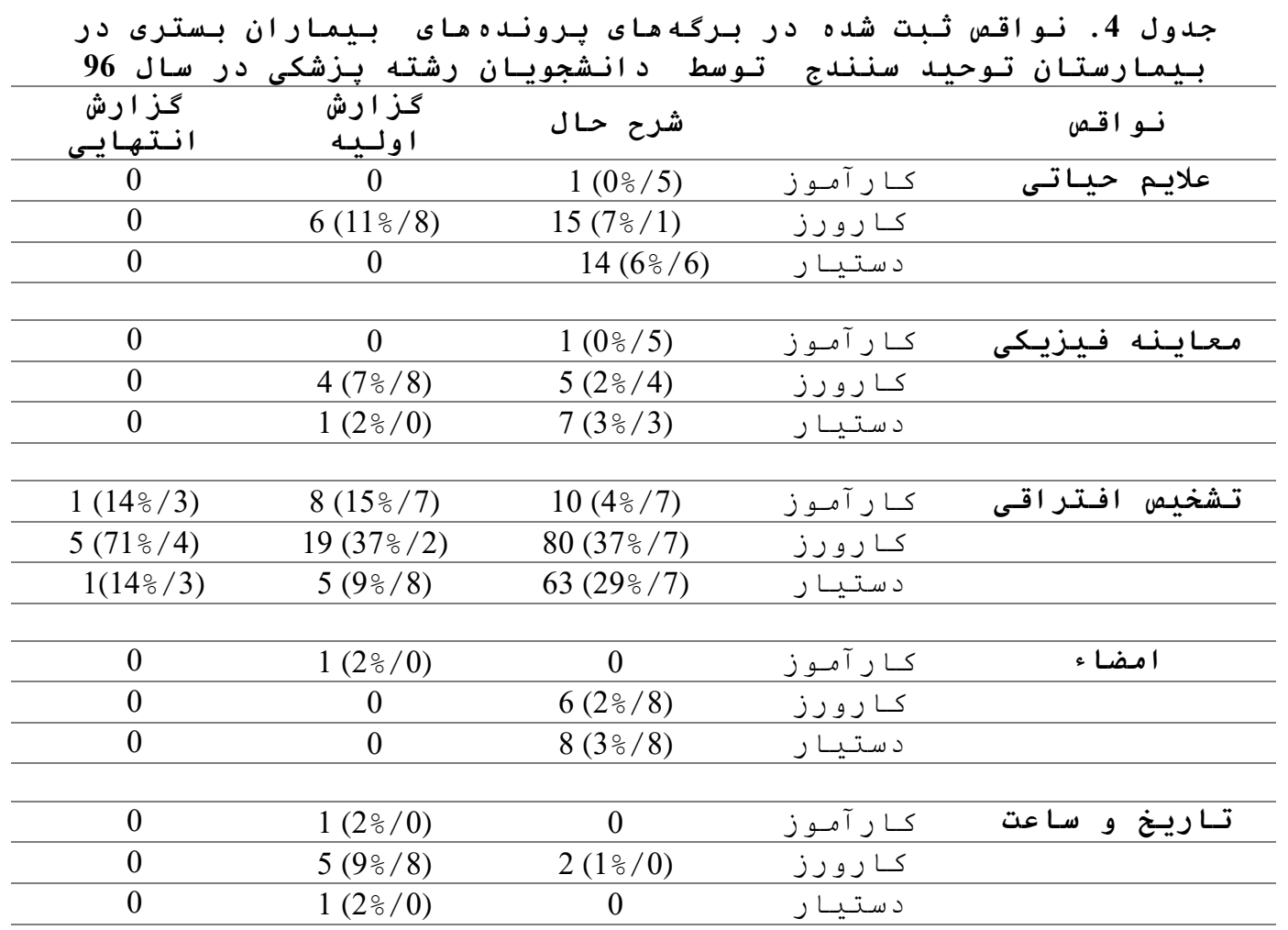

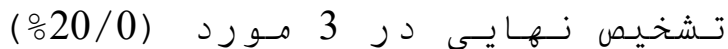

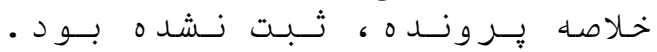

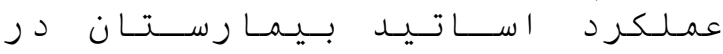

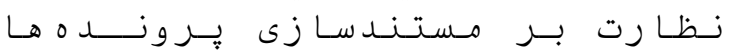

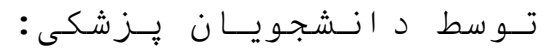

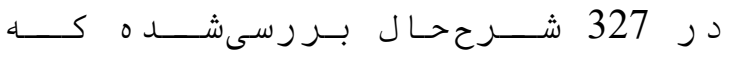

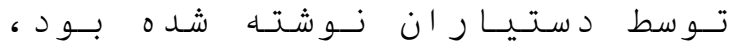

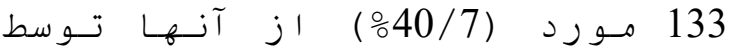

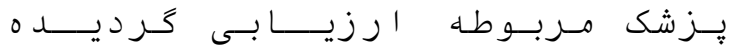

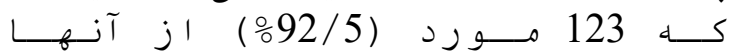

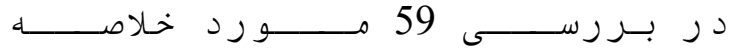

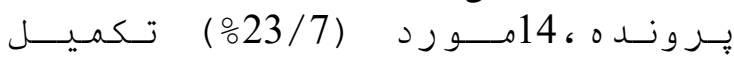

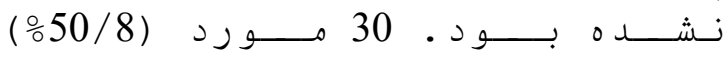

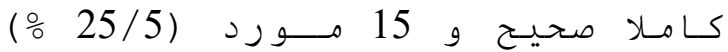

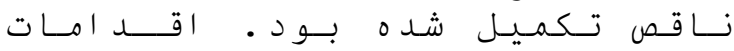

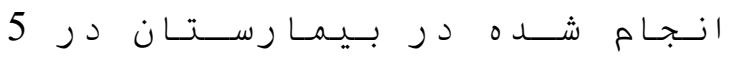

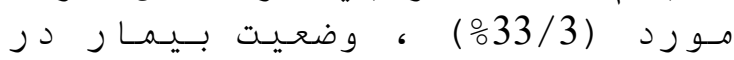

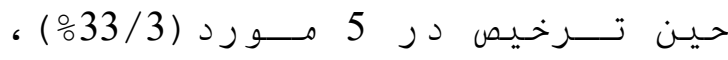

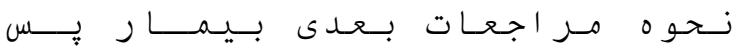

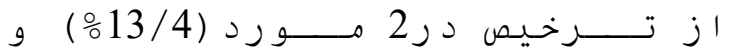


عدم ثـبـت سـاعت، 16 مـورود رد عدم ثـبـت

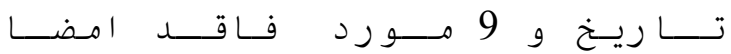

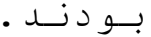

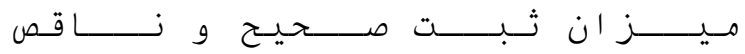

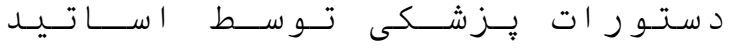

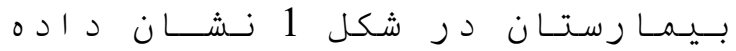

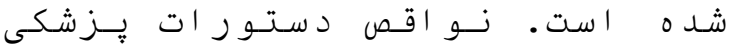

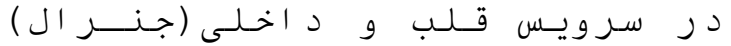

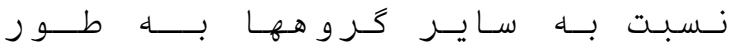

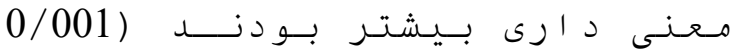

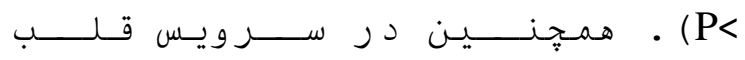

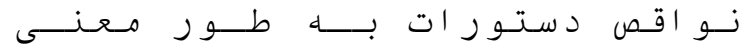

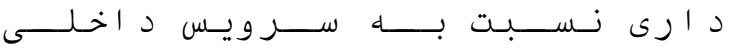

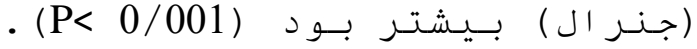

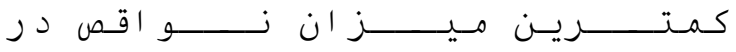

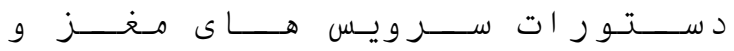

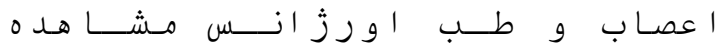

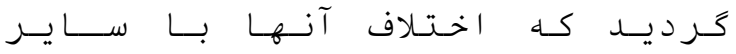

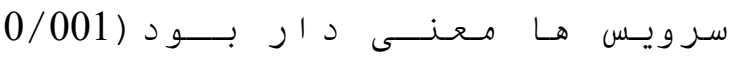

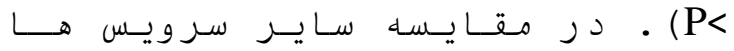

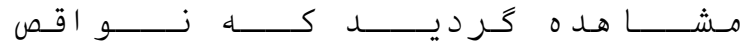

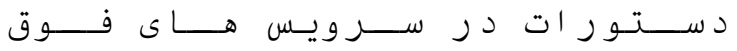

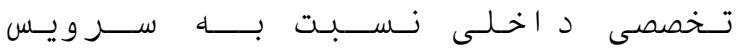

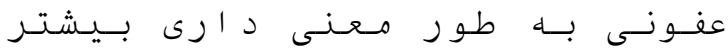
بـود د

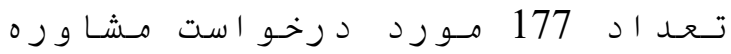

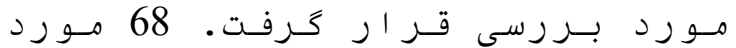

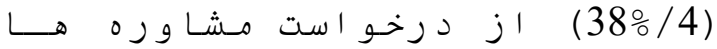

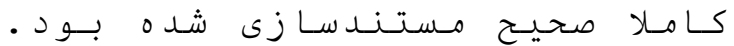

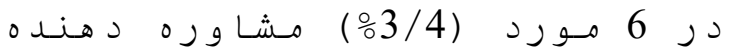

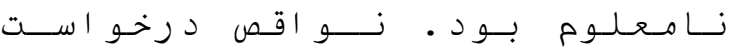

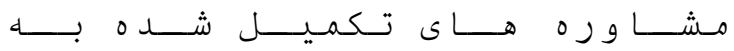

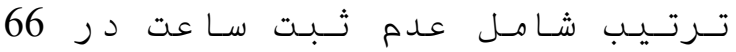

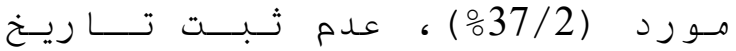

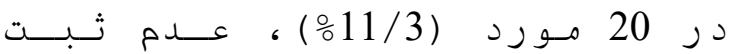

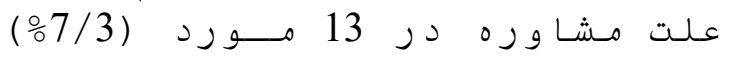

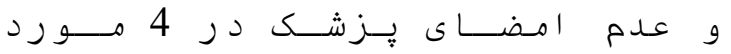

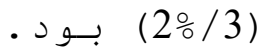

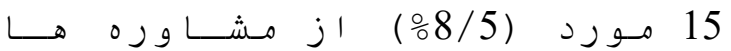

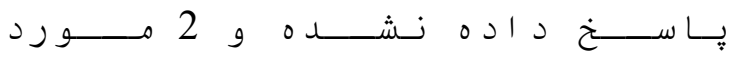
دا

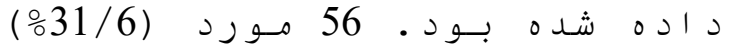

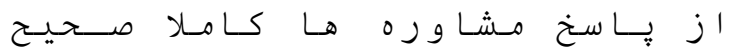

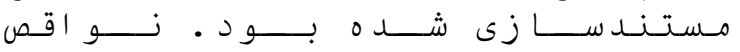

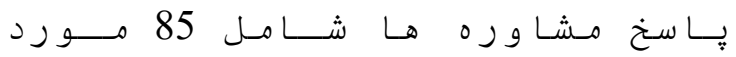

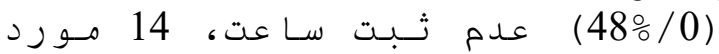

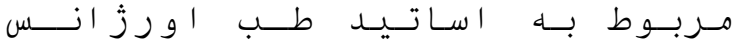

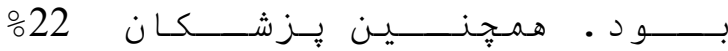

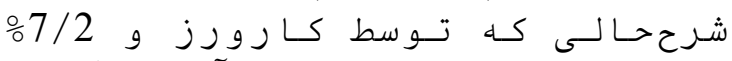

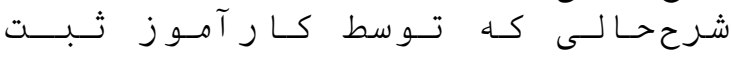

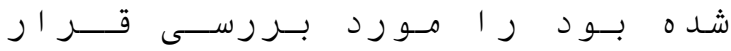

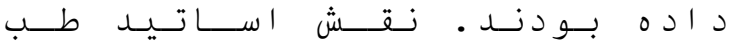

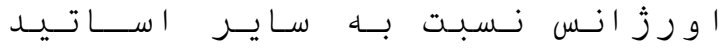

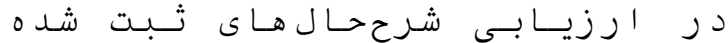

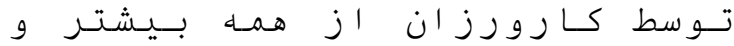

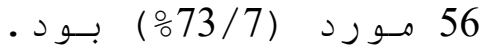

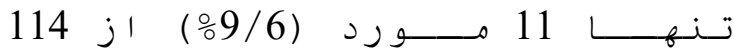

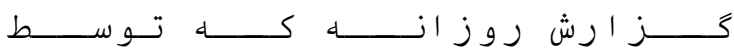

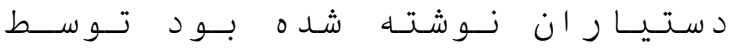

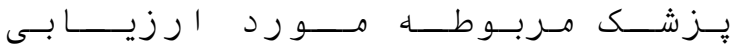

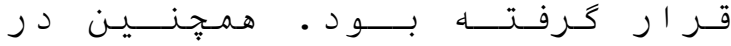

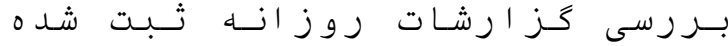

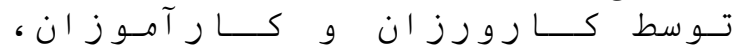

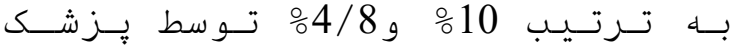

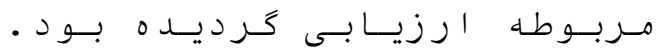

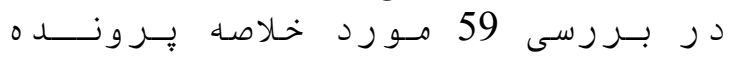

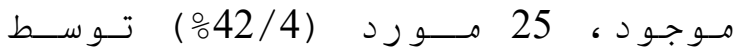

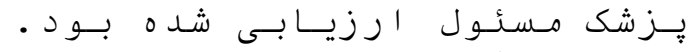

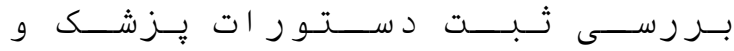

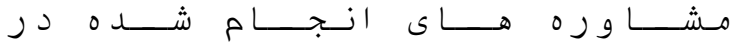

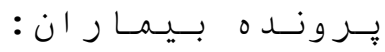

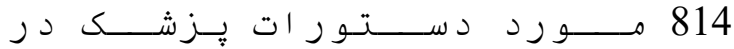

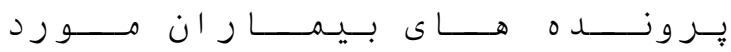

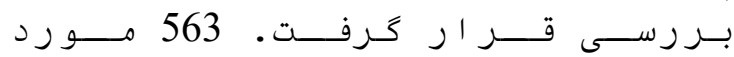

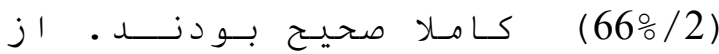

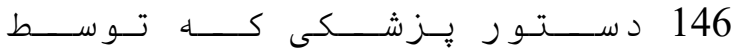

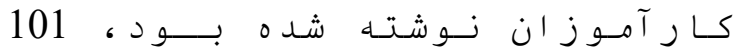

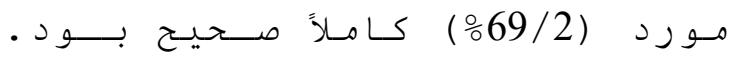

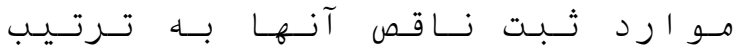

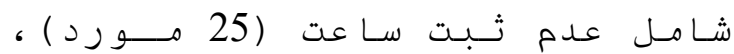

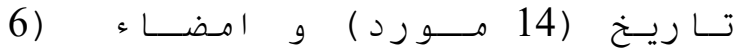

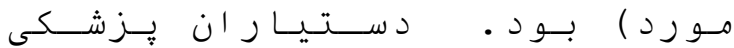

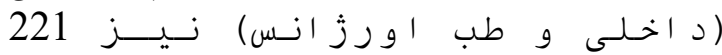

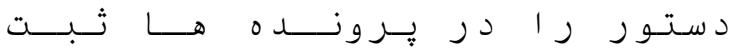

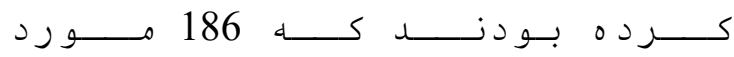
(87\%/7)

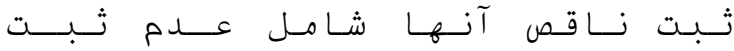

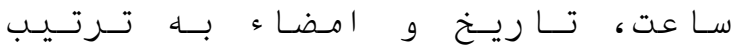

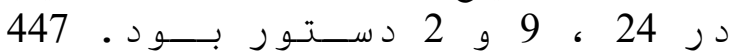

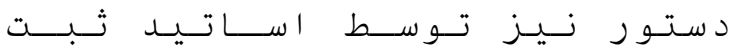

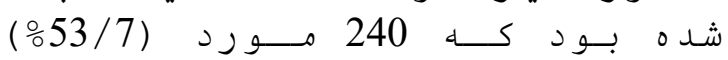

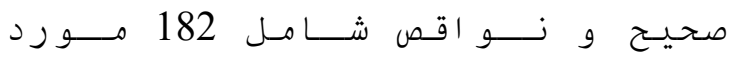




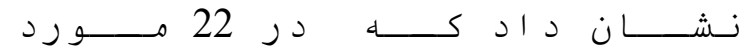

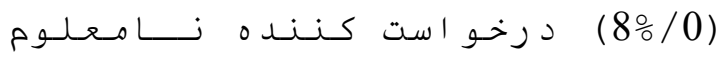

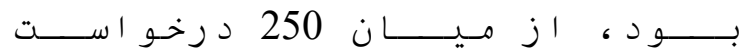

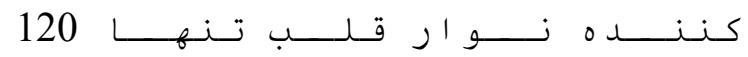

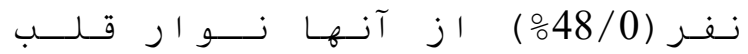

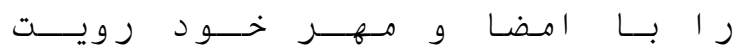

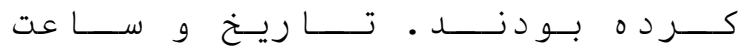

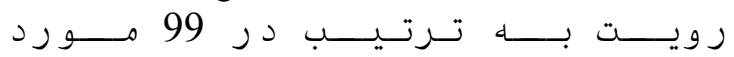

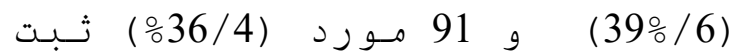

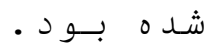

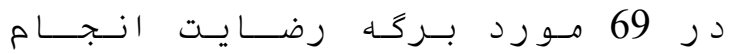

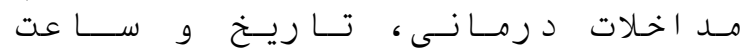

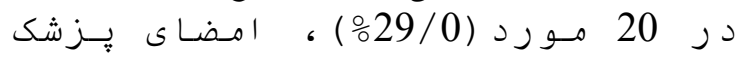

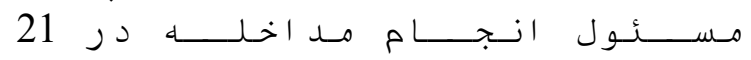

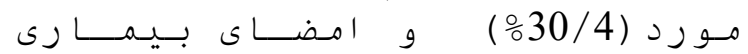

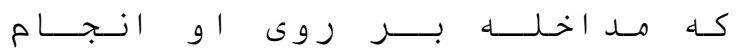

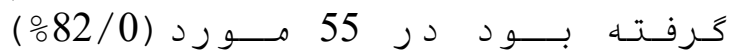

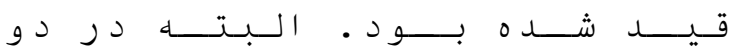

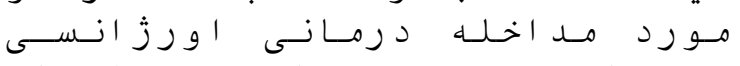

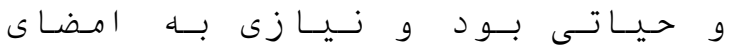

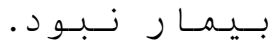

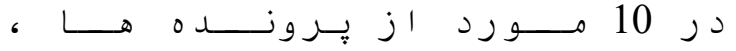

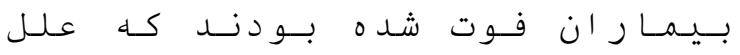

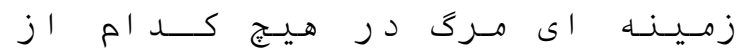

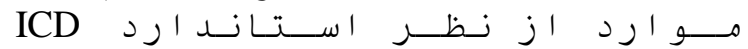

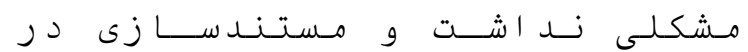

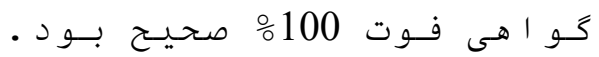

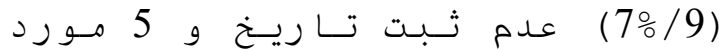
(2\%/8)

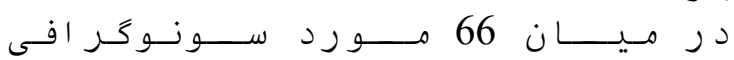

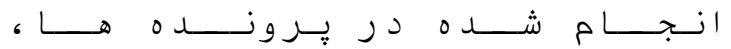

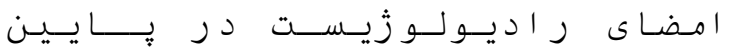

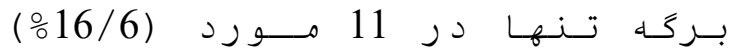

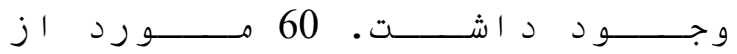

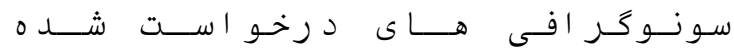

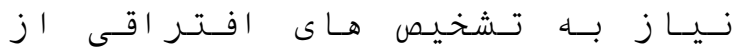

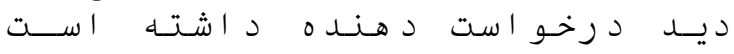

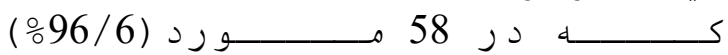

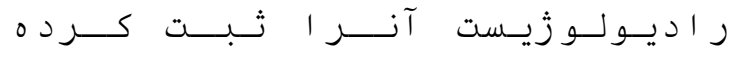

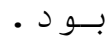

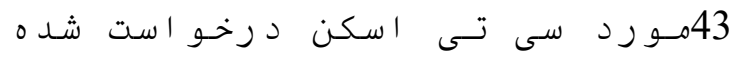

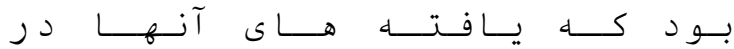

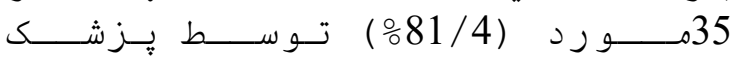

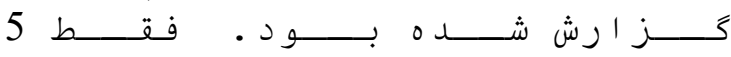

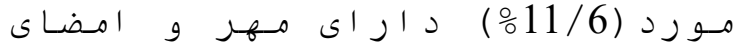

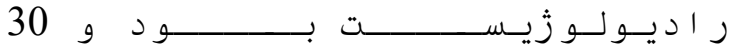

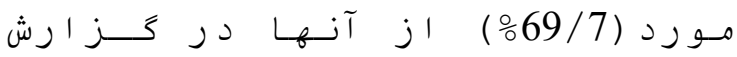

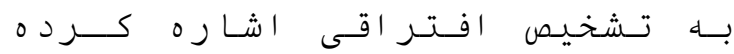

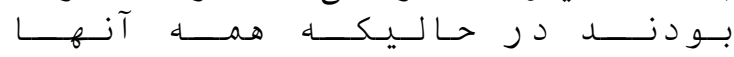

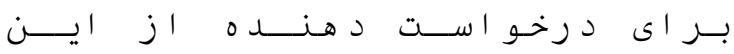

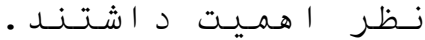

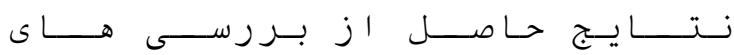

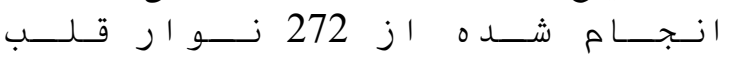

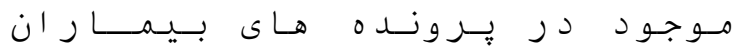

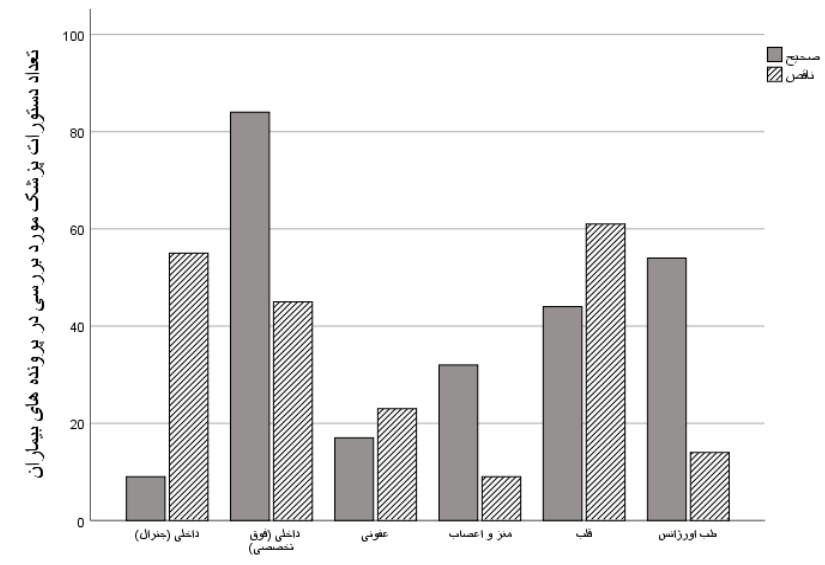

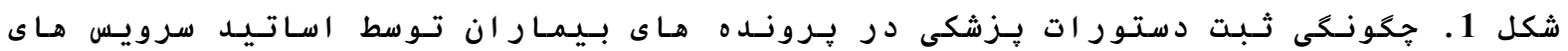

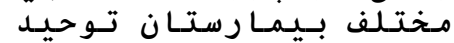

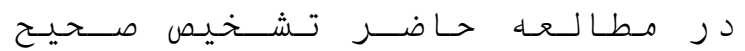

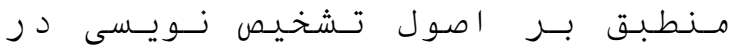




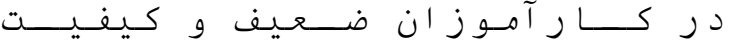

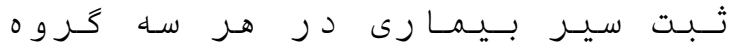

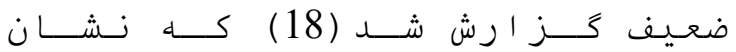

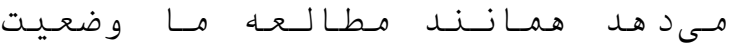

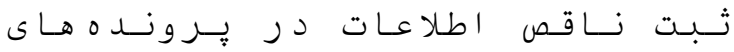

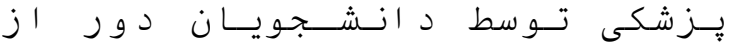

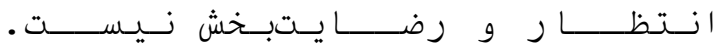

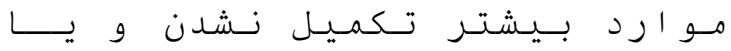

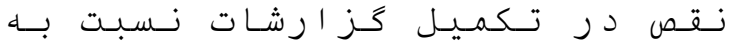

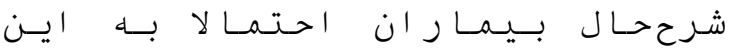

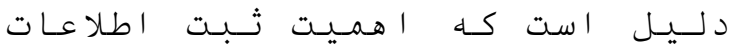

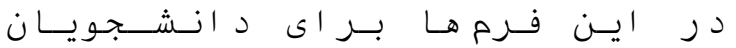

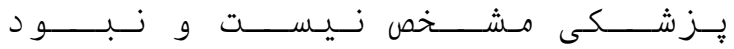

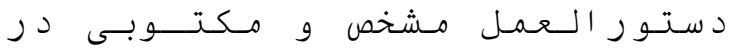

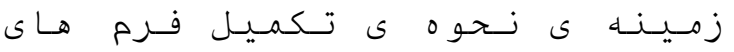

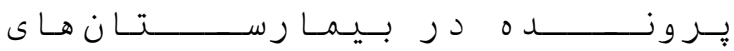

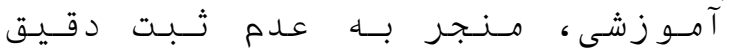

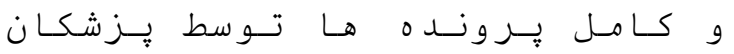

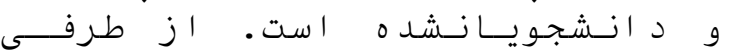

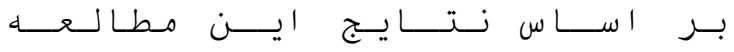

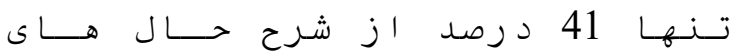

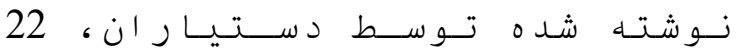

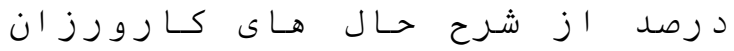

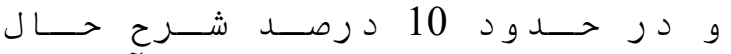

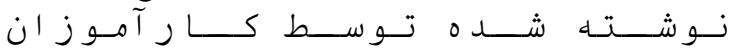

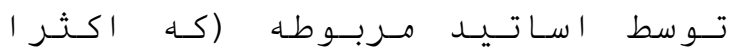

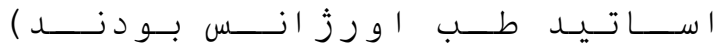

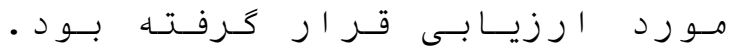

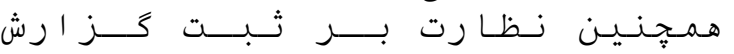

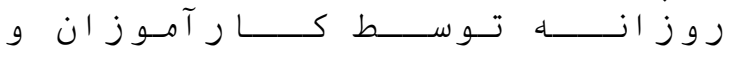

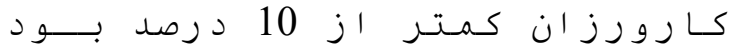

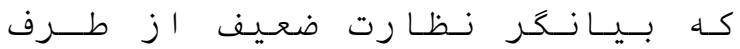

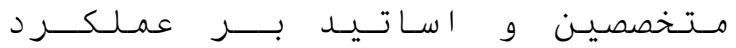

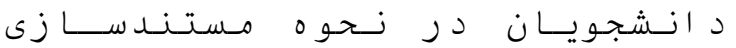

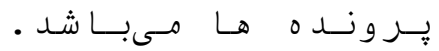

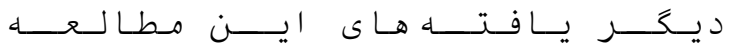

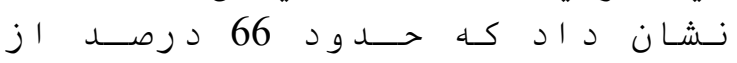

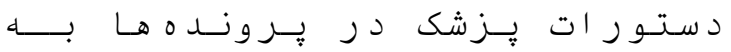

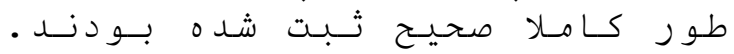

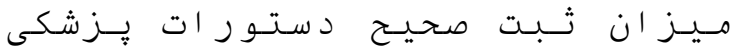

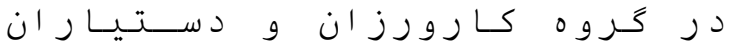

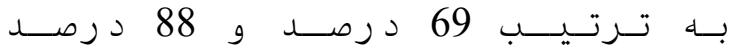

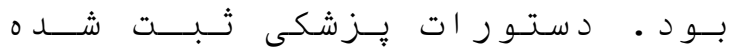

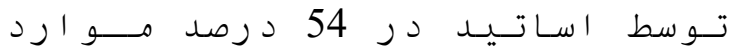

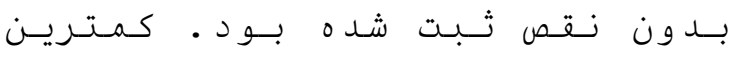

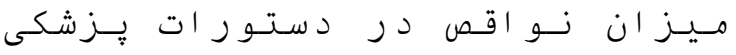

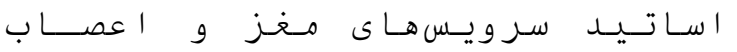

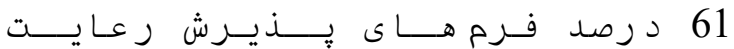

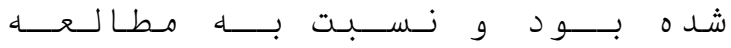

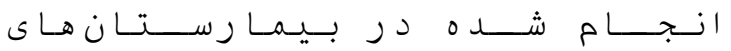

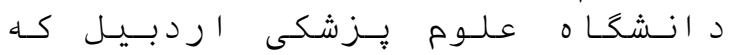

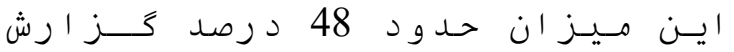

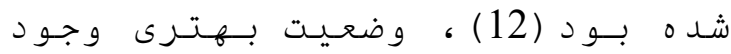

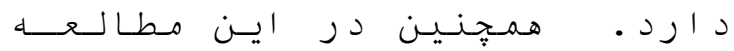

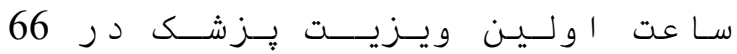

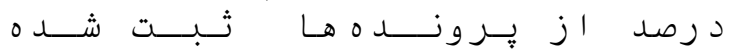

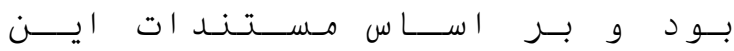

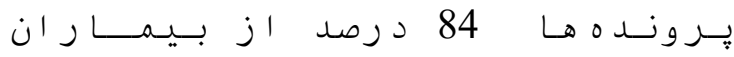

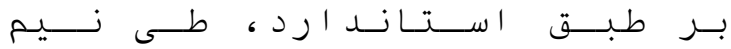

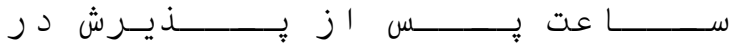

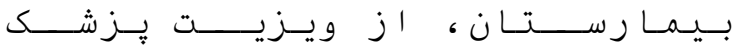

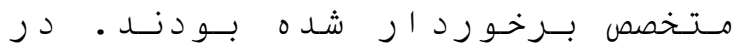

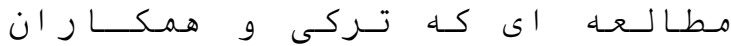

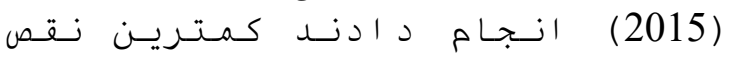

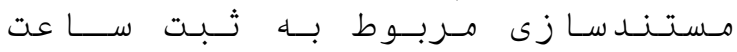

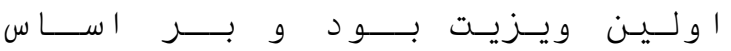

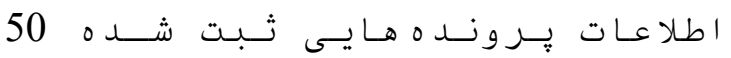

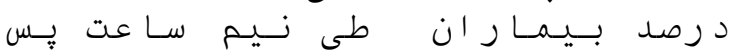

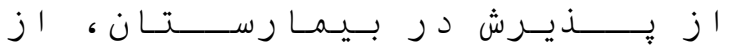

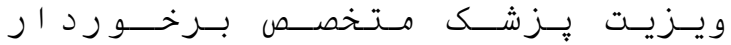

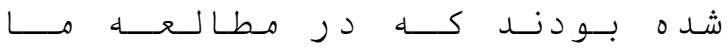

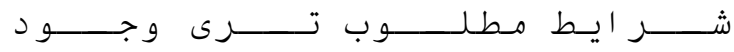

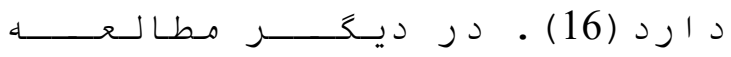

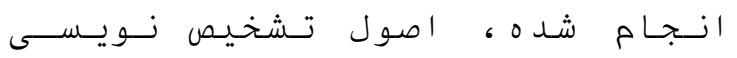

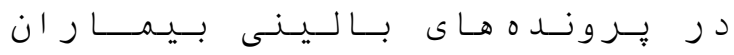

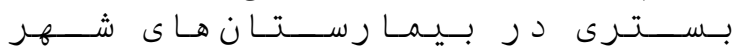

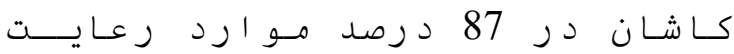

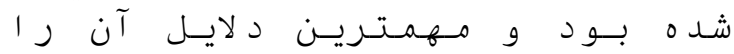

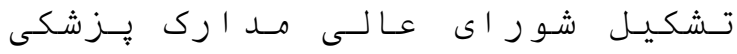

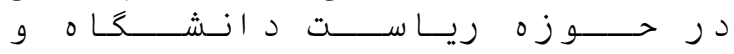

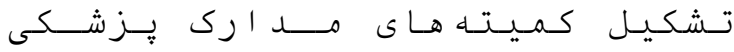

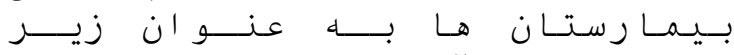

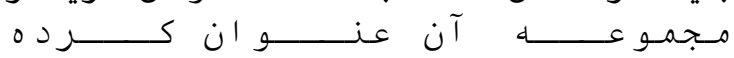

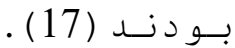

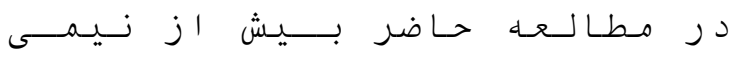

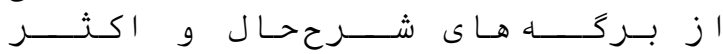

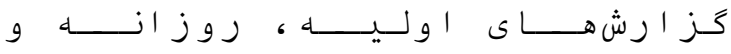

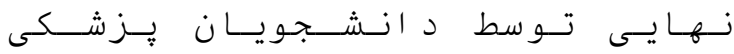

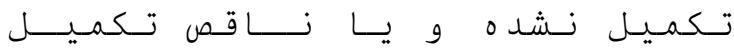

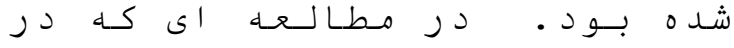

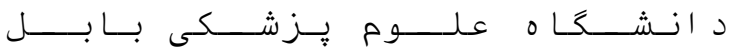

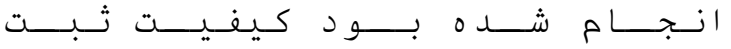

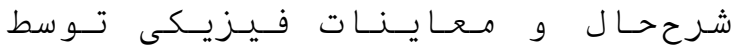

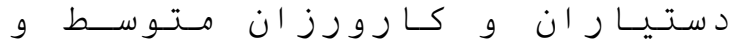




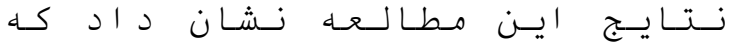

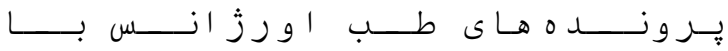

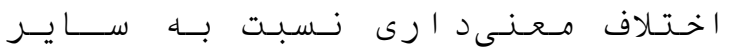

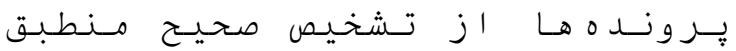

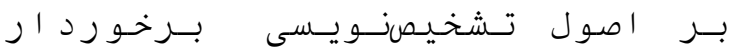

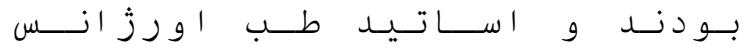

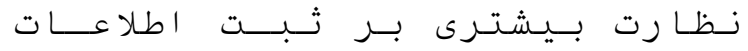

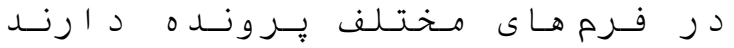

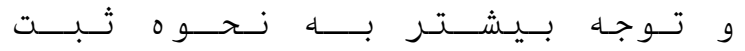

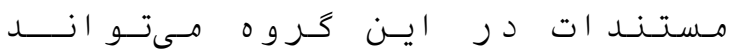

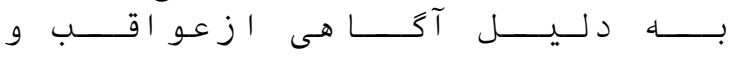

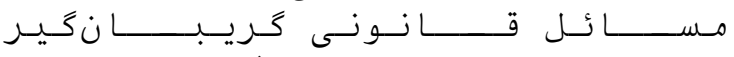

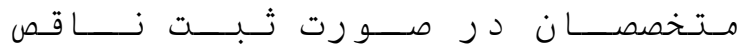

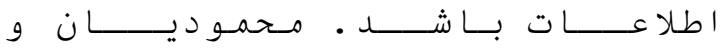

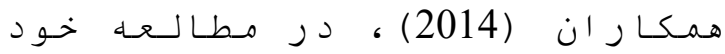

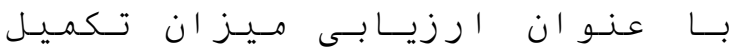

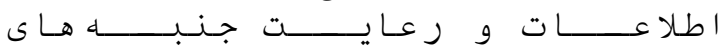

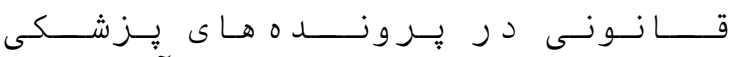

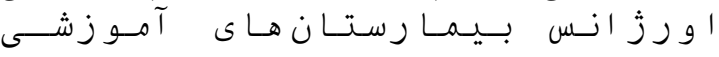

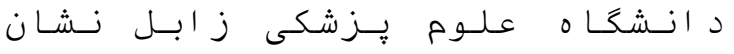

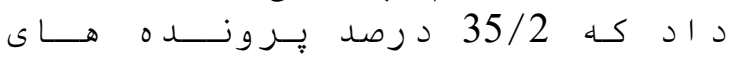

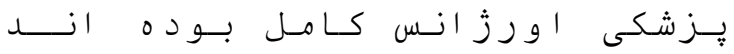

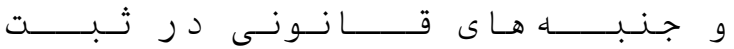

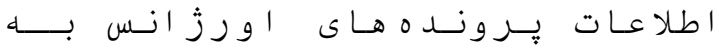

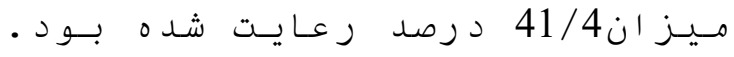

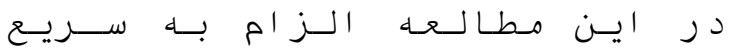

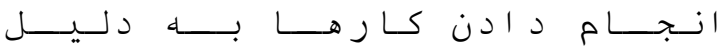

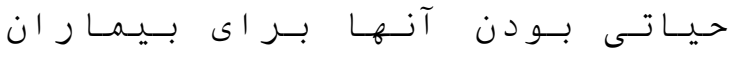

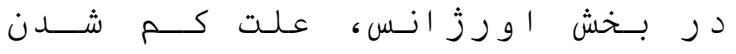

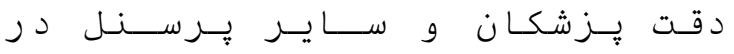

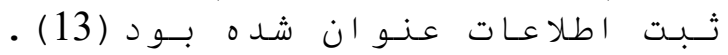

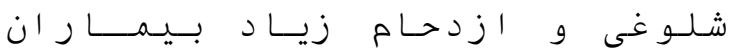

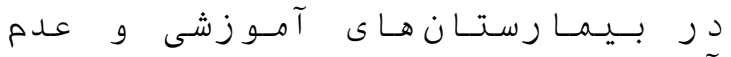

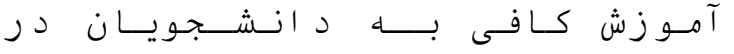

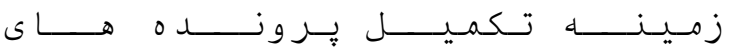

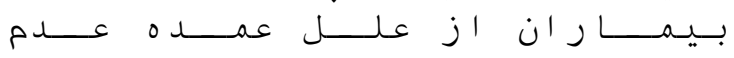

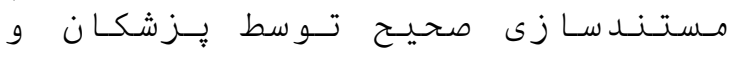

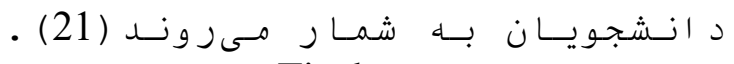

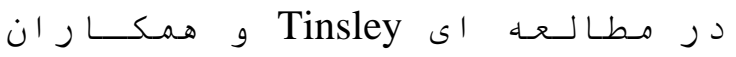

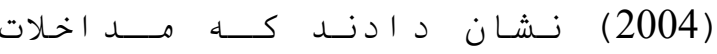

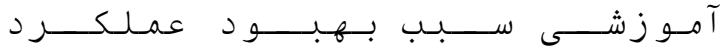

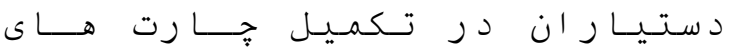

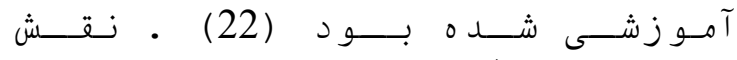

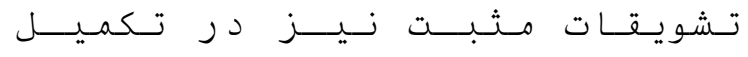

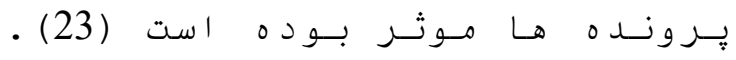

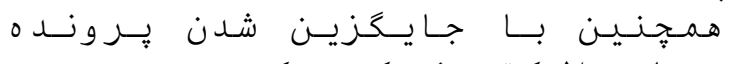

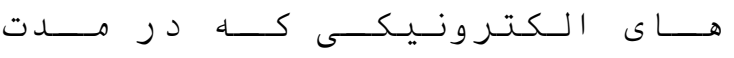

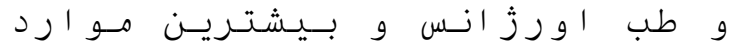

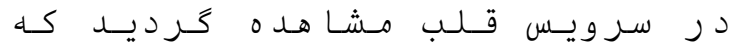

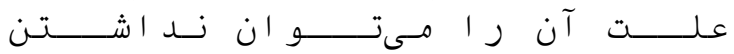

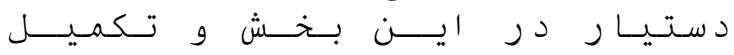

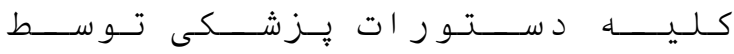

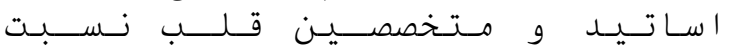

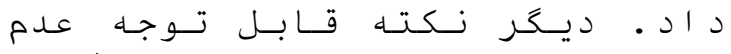

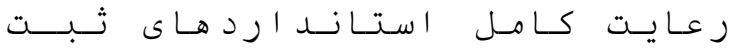

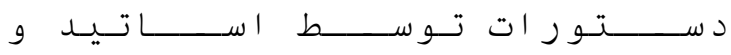

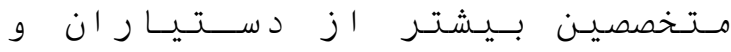

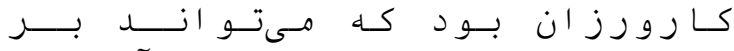

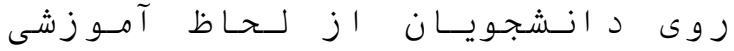

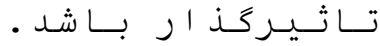

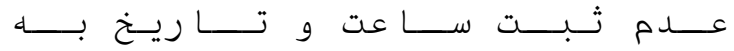

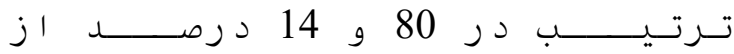

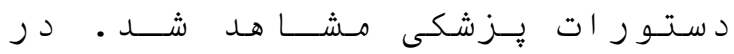

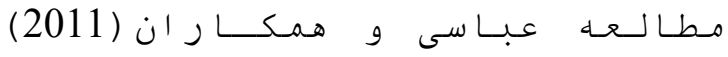

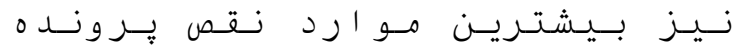

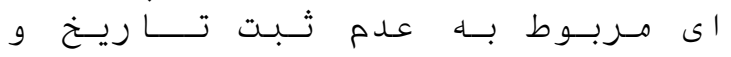

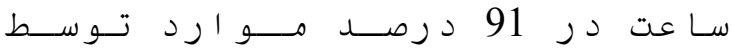

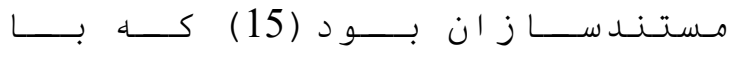

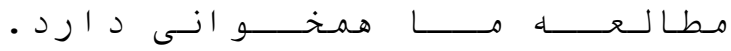

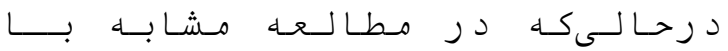

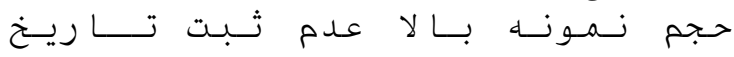

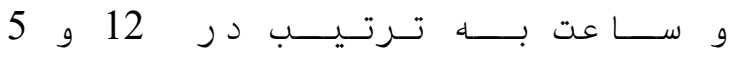

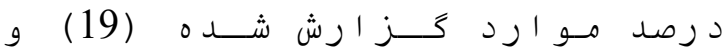

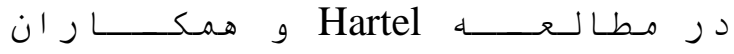

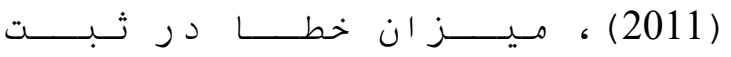

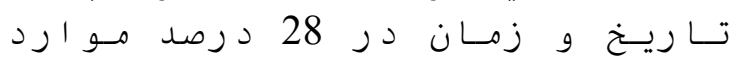

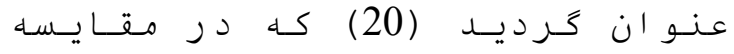

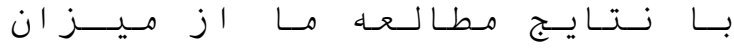

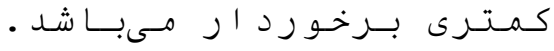

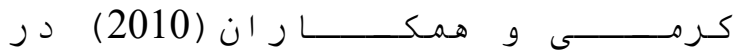

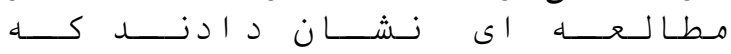

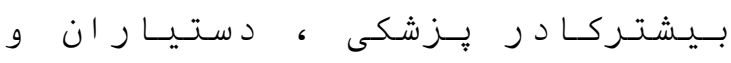

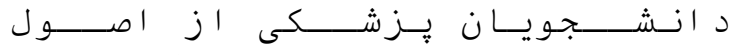

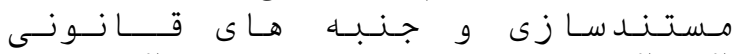

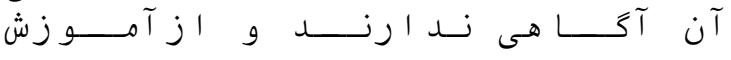

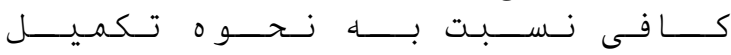

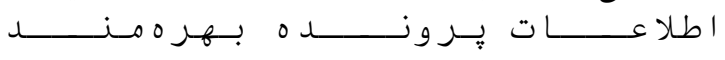

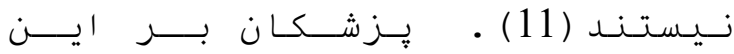

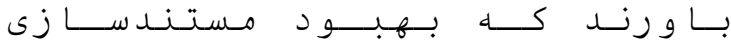

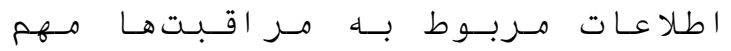

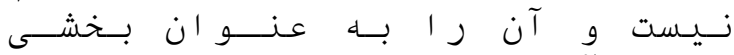

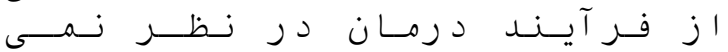

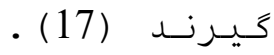




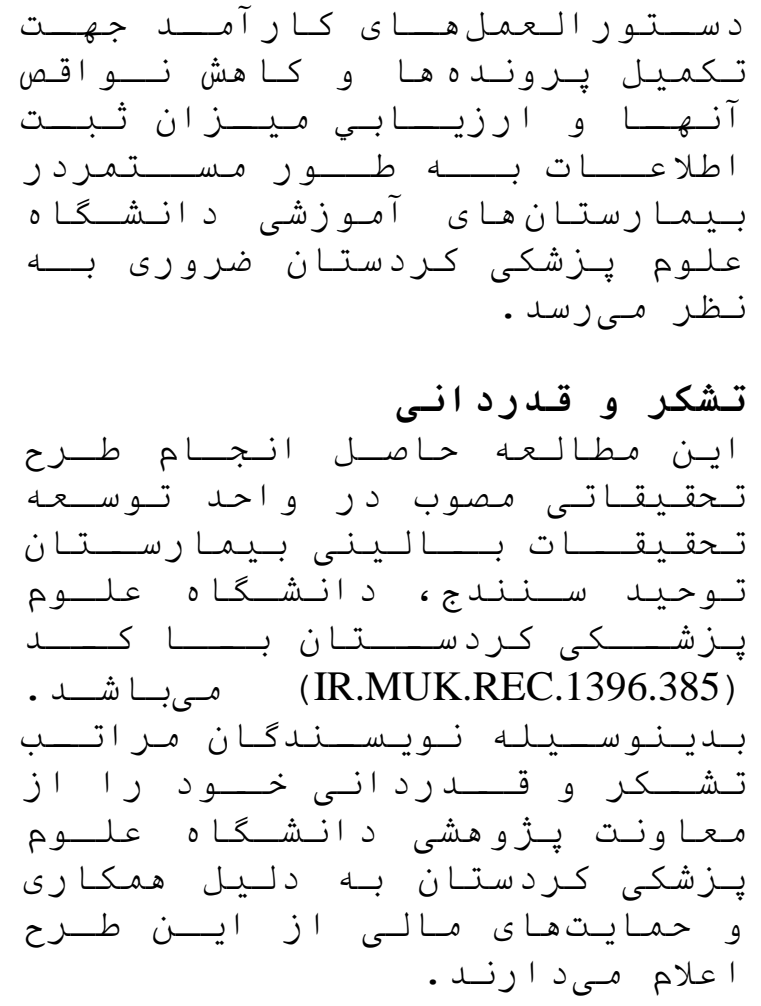

1.Standardization IOf. Quality Management Systems: Guidelines for Performance Improvements: International Organization for Standardization; 2000.

2.Askari H, Samandari A, Rastegari Z, Nadipour N. Identifying and documenting work processes promoting efficiency in emergency department ofShahid Mohammad Montazeri Hospital in 2012. RJMS. 2013;20(112):53-66.

3.Torki S, Tavakoli N, Khorasani E. Improving the Medical Record Documentation by Quantitative Analysis in a Training Hospital. J Environ Health Sci. 2015;1(1):22.

4.Alkureishi MA, Lee WW, Lyons M, Press VG, Imam S, Nkansah-Amankra A, et al. Impact of electronic medical record use on the patient-doctor relationship and communication: a systematicreview. J Gen Intern Med. 2016;31(5):548-60.

5.Mahfouzi A, Taghadosinzhad FAD, Abedi Khourasgani H. A 6 years study on anaesthesiologist's medical malpractice cases referred to Tehran medicolegal commission (1994-1999). J Forensic Med. 2002;8(26):4-10.

6.Liang BA. The adverse event of unaddressed medical error: identifying and filling the holes in the health-care and legal systems. JLME. 2001;29(3-4):346-68.

7.Liang BA. Risks of Reporting Sentinel Events: A system for reporting medical errors could be used for lawsuits rather than just for safety purposes. Health Aff. 2000;19(5):112-20.

8.Weng CY. Data accuracy in electronic medical record documentation. JAMA ophthalmology. 2017;135(3):232-33

9.Farzandipour M, Meidani Z, Rangraz Jeddi F, Gilasi H, Shokrizadeh Arani L, Mobarak, Zohreh.The Effect of Educational Intervention on Medical Diagnosis Recording among Residents in Kashan University of Medical Sciences. IJME. 2012;12(1):90-8.

10.Farzandipour M, Sadoughi F, Ahmadi M, Karimi I. Safety requirements for health electronic file; comparison between selected countries. 2007.1-9. 
11.Karami M, Shokrizadeh Arani L. Related factors in medical records documentation quality and presenting solutions from managers' and physicians' viewpoints occupied in hospitals affiliated to Kashan University of Medical Sciences. IJME. 2010;9(4):356-64.

12.Mashoufi M, Amani F, Rostami K, Mardi A. Evaluating Information Record in the Ardabil Medical Sciences University, 2002. JArUMS. 2004;4(1):43-9.

13.Mahmoudian S, Alidadi F, Arji G, Ramezani A. Evaluation ofCompleteness And Legal Aspects'compliance Of Emergency's Medical Records In Teaching Hospitals Of Zabol University Of Medical Sciences. J Paramed Sci. 2014;3(1):33-9.

14.Esmailian M, Nasr-Esfahani M, Brahimi A-S. The quality of patients' files documentation in Emergency Department; a cross sectional study. Iran. J Emerg Med. 2014;1(1):16-21.

15.Abbassi S, Tavakoli N. Quantitative analysis of medical record of patients admitted in the gharazi hospital. HIM. 2011;8(1):50-60.

16.Torki S, Tavakoli N, Khorasani E. Improving the Medical Record Documentation by Quantitative Analysis in a Training Hospital. J Environ Health Sci. 2015;1(1):22.

17.Farzandipour M, Asefzadeh S. Surveying The Principle Of Diagnosis Recording In Patients'Medical Records Of Kashan Hospitals In 1381. KAUMS Journal. 2004;8(3):36-44.

18.Esmaeili, MR, Abazari, H, Mohammadi Kenari H. Comparison of Medical Students and Pediatric Residents Practices in Medical Records at Amirkola Children Hospital. J Babol Univ Medical Sci. 2010;12(5):106-11.

19.Azimi 1, markazimoghaddam N, Rostami k, Talebi A, Eskandari A, Mirzaiy A, et al. Assessing the Physicians' Order Errors in Medical Records and it's effective Factors (A Case Study). Hospital. 2016;15(2):41-8.

20.Hartel MJ, Staub LP, Roder C, Eggli S. High incidence of medication documentationerrors in a Swiss university hospital due to the handwritten prescription process. BMC Health Serv Res. 2011;11:199.

21.Seif Rabiee MA, Sedighi I, Mazdeh M, Dadras F, Shokouhee Solgi M, Moradi A. Study of hospital records registration in teaching hospitals of Hamadan University of Medical Sciences in 2009. Avicenna J Clin Med. 2009;16(2):45-9.

22. Tinsley JA. An educational intervention to improve residents' inpatient charting. Academic psychiatry. 2004;28(2):136-9.

23.O'Leary KJ, Liebovitz DM, Feinglass J, Liss DT, Evans DB, Kulkarni N, et al. Creating a better discharge summary: improvement in quality and timeliness using an electronic discharge summary. J Hosp Med. 2009;4(4):219-25.

24.Kripalani S, LeFevre F, Phillips CO, Williams MV, Basaviah P, Baker DW. Deficits in communication and information transfer between hospital-based and primary care physicians: implications for patient safety and continuity of care. Jama. 2007;297(8):831-41.

25.Saravi BM, Asgari Z, Siamian H, Farahabadi EB, Gorji AH, Motamed N, et al. Documentation of Medical Records in Hospitals of Mazandaran University of Medical Sciences in 2014: a Quantitative Study. Acta Informatica Medica. 2016;24(3):202-6. 